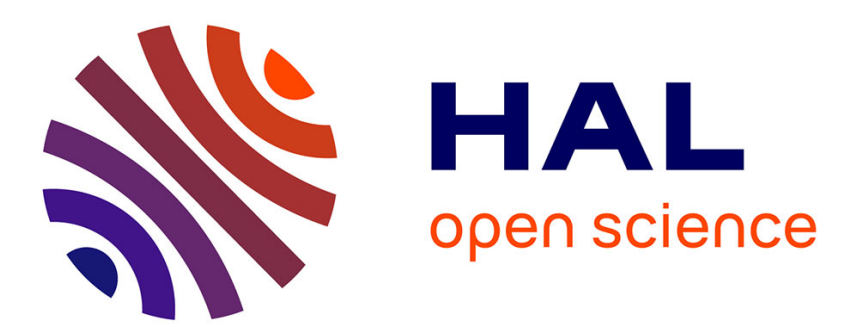

\title{
Selection Rules and Intensity Calculations for a Cs Asymmetric Top Molecule Containing a Methyl Group Internal Rotor
}

\author{
J.T. Hougen, I. Kleiner, M. Godefroid
}

\section{- To cite this version:}

J.T. Hougen, I. Kleiner, M. Godefroid. Selection Rules and Intensity Calculations for a Cs Asymmetric Top Molecule Containing a Methyl Group Internal Rotor. Journal of Molecular Spectroscopy, 1994, 163 (2), pp.559-586. 10.1006/jmsp.1994.1047 . hal-03360579

\author{
HAL Id: hal-03360579 \\ https://hal.science/hal-03360579
}

Submitted on 30 Sep 2021

HAL is a multi-disciplinary open access archive for the deposit and dissemination of scientific research documents, whether they are published or not. The documents may come from teaching and research institutions in France or abroad, or from public or private research centers.
L'archive ouverte pluridisciplinaire HAL, est destinée au dépôt et à la diffusion de documents scientifiques de niveau recherche, publiés ou non, émanant des établissements d'enseignement et de recherche français ou étrangers, des laboratoires publics ou privés. 
J. Mol. Spectrosc., 163, 559-586 (1994), doi : 10.1006/jmsp.1994.1047

\title{
Selection Rules and Intensity Calculations for a $C_{s}$ Asymmetric Top Molecule Containing a Methyl Group Internal Rotor
}

\author{
Jon T. Hougen, ${ }^{*}$ 1. KLEINER, ${ }^{\dagger}{ }^{1}$ AND M. Godefroid $\ddagger^{2}$ \\ * Molecular Physics Division. National Institule of Standards and Technology, Gaithershurg, Maryland \\ 20899; † Laboratoire de Physique Moléculaire et Applications, Université Pierre et Marie Curie et \\ C.N.R.S. 4 Place Jussieu, Tour 13, 75252 Paris Cedex 05, France; and $\ddagger$ Laboratoire \\ de Chimie Physique Moléculaire-CPI60/09. Université Libre de Bruxelles. \\ Avenue F. D. Roosevelt, 50, 1050 Bruxelles, Belgium
}

\begin{abstract}
A detailed discussion is presented of the relationships between four different molecular symmetry groups, i.e., $D_{2}, C_{\mathrm{s}}, C_{3 v}$, and $C_{3 \mathrm{v}}^{(m)}$, commonly used to discuss energy level symmetry species and electric-dipole selection rules for internal rotor problems in molecules with a symmetric rotor top and a frame with a plane of symmetry, paying particular attention to confusion arising from the fact that the smaller groups are not always subgroups of the larger, and the groups are applied to different parts of the internal rotation problem (i.e., $D_{2}$ to pure rotation, $C_{s}$ to vibration-rotation, $C_{3 v}$ and $C_{3 \mathrm{v}}^{(m)}$ to torsion-rotation). The meaning in this context of the traditional $K_{a}, K_{c}$ labels for rotational energy levels in molecules with internal rotation is examined in detail. A discussion is also given of the relationship between three different schemes for defining a molecule-fixed axis system in internal rotation problems, i.e., the principal axis method, the internal axis method, and a hybrid method referred to here as the "rho axis method," paying particular attention to the meaning of $a$-type, $b$-type, and $c$-type transitions when using each of these axis systems. Some of the above ideas were helpful in adding intensity calculations to our earlier computer program treating internal rotation and overall rotation in acetaldehyde-like molecules. and these intensity
\end{abstract} calculations are also brielly discussed.

\section{INTRODUCTION}

In spectroscopic studies of an asymmetric top molecule containing an internal rotor group it is not uncommon to use a mixture of language and formalism arising from a rigid-rotor model, a traditional vibration-rotation model, and an internal rotor model. In this paper we discuss some aspects of the confusion which can arise from this simultaneous use of physical ideas from three rather different models. To simplify an already complicated situation, we limit consideration to an asymmetric rotor molecule consisting of a symmetric rotor top which has $C_{3 v}$ symmetry and a frame which has a plane of symmetry. This class of molecules contains methanol, propene, acetaldehyde, etc.

In Section 2 we discuss four molecular symmetry groups and their range of application, namely $D_{2}$ for pure rotation, $C_{s}$ for vibration-rotation, and $C_{3 v}$ or $C_{3 v}^{(m)}$ for torsion-rotation (or vibration-torsion-rotation), paying particular attention to the use of asymmetric rotor $K_{a}, K_{c}$ labels for the rotational states and to the use of $a$-type, $b$-type, and $c$-type labels for electric-dipole transitions.

In Section 3 we discuss three different molecular axis systems in common use for treating the torsion-rotation problem, namely a system (PAM) locked to the principal

\footnotetext{
' NIST Guest Worker. 1989-1991.

${ }^{2}$ Senior Research Associate of the Belgian National Fund for Scientific Research (FNRS).
} 
inertial axes, a system (RAM) locked in the frame with the $z$ axis parallel to a vector often called $\rho$ in the literature, and a system (IAM) "floating" with respect to both the frame and the top, in which torsional angular momentum vanishes. Details of the form of the Hamiltonian, and hence details of the $K_{a}, K_{c}$ labeling and of the $a, b, c$ type labeling, depend on which of these three axis systems is used.

In Section 4 we return to the question of $a$-type, $b$-type, and $c$-type transitions for the RAM and IAM systems. We also describe a computer program for calculating intensities in the RAM system.

\section{GROUP THEORY AND SYMMETRY LABELS}

A question that soon arises, when large-amplitude motions are present in a molecule, is which symmetry group to work with. In fact, four different symmetry groups (i.e., $D_{2}, C_{s}, C_{3 \mathrm{v}} \equiv G_{6}$ and $\left.G_{6}^{(m)}\right)$, and therefore four different ways of labeling the energy levels, have been used in discussing vibration-rotation-torsion (vrt) energy levels in molecules of the type under discussion here. In an attempt to eliminate some of the confusion arising from the simultaneous use of these four groups, we consider the limiting cases of applicability and the symmetry operations for each of these groups in turn.

\section{Limiting Case (r) with Point Group $\mathrm{D}_{2}$}

This limiting case arises when only overall rotation $(r)$ is considered. The traditional group-theoretical treatment of rotational energy levels for all asymmetric top molecules (1), regardless of the symmetry properties of their equilibrium geometry, corresponds to use of the point group $D_{2}$, which contains the four symmetry operations $E, C_{2}(a)$, $C_{2}(b)$, and $C_{2}(c)$, where $C_{2}$ represents a twofold rotation, and $a, b, c$ represent the three mutually perpendicular principal axes of inertia of the asymmetric top. The well-known character table of this group is given in Table I.

TABLE I

\begin{tabular}{|c|c|c|c|c|c|c|}
\hline \multicolumn{7}{|c|}{ Character Table of $D_{2}$} \\
\hline$\Gamma$ & $k_{a} k_{c}$ & $\mathbf{E}$ & $C_{2}(a)$ & $C_{2}(b)$ & $c_{2}(c)$ & Operator ${ }^{\mathrm{b}}$ \\
\hline$A$ & ee & 1 & 1 & 1 & 1 & $\mu_{a}, \mu_{b}, \mu_{c}$ \\
\hline $\mathrm{B}_{\mathbf{a}}$ & eo & 1 & 1 & -1 & -1 & $\mathrm{~J}_{\mathbf{a}}, \Phi_{\mathrm{Za}}$ \\
\hline $\mathbf{B}_{\mathbf{b}}$ & oo & 1 & -1 & 1 & -1 & $\mathrm{~J}_{\mathrm{b}}, \Phi_{\mathrm{Zb}}$ \\
\hline $\mathrm{B}_{\mathrm{C}}$ & oe & 1 & -1 & -1 & 1 & $J_{c}, \Phi_{Z c}$ \\
\hline \multicolumn{7}{|c|}{$\begin{array}{l}\text { aThe species } \Gamma \text { are one of several notations } \\
\text { used; e(ven)o(dd) labels for } \mathrm{K}_{\mathrm{a}} \mathrm{K}_{\mathrm{c}} \text { are common } \\
\text { asymmetric rotor notation. } \\
\mathrm{b}_{\text {Molecule-fixed components of } \mathrm{J} \text { and components }} \\
\Phi_{\mathrm{Za} \text {, etc. of the direction cosine matrix are }} \\
\text { defined on pp. } 282 \text { and } 286 \text { of Ref. ( } 2) \text {, with } \\
\mathrm{a}, \mathrm{b}, \mathrm{c} \rightarrow \mathrm{z}, \mathrm{x}, \mathrm{y} \text {. Molecule-fixed components of } \\
\text { the dipole moment operator } \mu \text { are independent } \\
\text { of the Euler angles and are therefore } \\
\text { invariant to the operations of this group. }\end{array}$} \\
\hline
\end{tabular}


Although it is not always explicitly stated, the effect of these symmetry operations can be defined for many asymmetric tops only for the three Eulerian angles describing the overall rotational motion of the molecule (as is done, for example, in Appendix I of Ref. (2)). A given $C_{2}$ operation has vibrational significance only when it can move every vibrational displacement vector from its original atom to an identical atom located at a position defined by rotating the original atom through $180^{\circ}$ about the $C_{2}$ axis. This will only be the case when the molecule has an equilibrium geometry such that this $C_{2}$ operation belongs to the point group of the molecule.

Transformation properties of the Eulerian angles under the operations of $D_{2}$ with $a, b, c \rightarrow z, x, y$ are given in Table II. Transformation properties of the moleculefixed components of the total angular momentum operator (2) derived from the coordinate transformations in Table II are given in Table I.

The reason why $D_{2}$ is useful for all asymmetric top molecules arises from the fact that the effective rotational Hamiltonian $H_{\mathrm{r}}$ for any isolated asymmetric top molecule can be expanded, as is well known (3-5), in a series of products containing even powers of the three components of the molecule-fixed angular momentum components; e.g.,

$$
\begin{array}{r}
H_{\mathrm{r}}=A J_{a}^{2}+B J_{b}^{2}+C J_{c}^{2}-D_{J}\left(\mathbf{J}^{2}\right)^{2}-D_{J K} J_{a}^{2} \mathbf{J}^{2}-D_{K} J_{a}^{4}-2 \delta_{J} \mathbf{J}^{2}\left(J_{b}^{2}-J_{c}^{2}\right) \\
-\delta_{K}\left[J_{a}^{2}\left(J_{b}^{2}-J_{c}^{2}\right)+\left(J_{b}^{2}-J_{c}^{2}\right) J_{a}^{2}\right]+\text { higher order terms. }
\end{array}
$$

It can be seen from Table I that all the operators in $H_{\mathrm{r}}$ (and all higher order terms containing even powers of each angular momentum component) are totally symmetric under the operations of $D_{2}$. This implies that the symmetry species of $D_{2}$ can be used to label all eigenvalues (rotational energy levels) obtained from $H_{\mathrm{r}}$ in Eq. (1).

Rather than use the species $A, B_{a}, B_{b}$, and $B_{c}$ (or some other notation for the irreducible representations of $D_{2}$ ), spectroscopists normally prefer to use instead the two labels $K_{a}$ and $K_{c}$, where from a purely formal group-theoretical point of view $(-1)^{K_{a}}$, and therefore the parity of $K_{a}$, gives the +1 or -1 character in the $C_{2}(a)$ column of Table I, and $(-1)^{K_{c}}$ gives the character in the $C_{2}(c)$ column. (Characters for $C_{2}(b)$ need not be specified since they are simply the product of those for $C_{2}(a)$ and $C_{2}(c)$.) Since the prolate (or oblate) symmetric top basis functions used to set up the asymmetric rotor Hamiltonian matrix contain a factor $e^{+i K_{a} x}\left(\right.$ or $e^{+i K_{c} x}$ ), and since $C_{2}(a)$ for $a, b, c \rightarrow z, x, y$ (or $C_{2}(c)$ for $\left.a, b, c \rightarrow x, y, z\right)$ corresponds to $\chi \rightarrow$ $\chi+\pi$, the rigorous, but purely formal, group-theoretical characters also conveniently permit a physical interpretation, i.e., the parity of $K$.

TABLE II

Transformation Properties in $D_{2}{ }^{\mathbf{a}}$ of the Eulerian Angles ${ }^{b}$

$$
\begin{aligned}
E f(x, \theta, \phi) & =f(x, \theta, \phi) \\
C_{2}(a) f(x, \theta, \phi) & =f(\pi+x, \theta, \phi) \\
C_{2}(b) f(x, \theta, \phi) & =f(-x, \pi-\theta, \pi+\phi) \\
C_{2} \text { (c) } f(x, \theta, \phi) & =f(\pi-x, \pi-\theta, \pi+\phi)
\end{aligned}
$$

\footnotetext{
a See Table I.

$b_{f}(x, \theta, \phi)$ represents an arbitrary

function of these angles.
} 
Selection rules for electric dipole transitions can be determined $(I)$ by considering transformation properties of the laboratory-fixed $Z$ component of the electric dipole moment operator,

$$
\mu_{Z}=\Phi_{Z x} \mu_{x}+\Phi_{Z y} \mu_{y}+\Phi_{Z z} \mu_{z} .
$$

Since the direction cosine matrix elements $\Phi$ are functions only of the Eulerian angles (2), their transformation properties can be determined from Table II. Species of the three direction cosines occurring in Eq. (2) for $a, b, c \rightarrow z, x, y$ are shown in Table I. The three molecule-fixed components of the electric-dipole moment operator $\mu$ occurring on the right of Eq. (2) are constants within the framework of the purely rotational problem (i.e., independent of the Euler angles), and thus remain unchanged under the symmetry operations of $D_{2}$. As a result, the laboratory-fixed $Z$ component of $\mu$ does not have a well-defined symmetry species. Although this difficulty with the symmetry species of $\mu_{/}$, will disappear when we consider vibration-rotation symmetry properties, it is nevertheless possible to determine selection rules for purely rotational transitions from the species of $\Phi_{z x}, \Phi_{Z y}$, and $\Phi_{z z}$. Examination of Table I, together with the relation between $K_{a}, K_{c}$ and the characters for $C_{2}(a), C_{2}(c)$, indicates that transitions induced by $\mu_{a}$ (and therefore involving matrix elements of $\Phi_{Z z}$ for $a, b$, $c \rightarrow z, x, y$ ) obey $\Delta K_{a}, \Delta K_{c}=$ even, odd; transitions induced by $\mu_{h}$ obey $\Delta K_{a}, \Delta K_{c}$ $=$ odd, odd; and transitions induced by $\mu_{c}$ obey $\Delta K_{a}, \Delta K_{c}=$ odd, even. It is thus clearly possible to define four mutually exclusive types of pure rotational transitions in an asymmetric top, namely (i) $\Delta K_{a}, \Delta K_{c}=$ even, odd, (ii) $\Delta K_{a}, \Delta K_{c}=$ odd, odd, (iii) $\Delta K_{a}, \Delta K_{c}=$ odd, even, and (iv) $\Delta K_{a}, \Delta K_{c}=$ even, even, which are then conveniently referred to as $a$-type, $b$-type, $c$-type, and forbidden transitions, respectively.

This concludes our brief discussion of limiting case $(r)$, but in preparation for later limiting cases, we note that operators of the form $J_{a} J_{b}, \pi_{a} J_{a}$, or $p_{\alpha} J_{a}$, where $\pi$ is the vibrational angular momentum (2) and $p_{t r} \equiv-i \hbar \partial / \partial \alpha$ is the internal rotation angular momentum ( $\alpha$ is the torsional angle), would not be totally symmetric in the group $D_{2}$. The operator $J_{a} J_{b}$ is of species $B_{c} \neq A$ in Table $\mathrm{I}$; transformation properties of $\pi$ and $p_{\alpha}$ are not defined at all in Table II.

\section{Limiting Case (vr) with Point Group C,}

This limiting case is appropriate when small-amplitude vibrations $(v)$ and overall rotation $(r)$ are considered together $(v r)$. The traditional group-theoretical treatment of vibration-rotation energy levels when large-amplitude vibrations are absent $(2,6)$ involves use of the point group to which the equilibrium geometry of the molecule belongs. In the present paper, this point group is by hypothesis $C_{s}$, which contains only two elements ( $E$ and $\sigma$ ). Table III gives the symmetry species and characters for $C_{s}$.

The fact that the vibration-rotation point group here (and in general) is not a subgroup of the group $D_{2}$ used for the purely rotational problem prevents any rigorous mathematical use of group-theoretical correlation tables, etc., when passing from the pure rotational to the vibration-rotation problem.

Symmetry operations in $C_{s}$ are defined for both the small-amplitude vibrational displacement variables $\mathbf{d}_{i}(2)$ and the Eulerian angles $\chi \theta \phi(2)$ describing the overall rotation of the molecule. These vibrational and rotational variables can in turn be precisely defined, as explained in more detail elsewhere $(7,8)$, in terms of the laboratory-fixed coordinates $\mathbf{R}_{i}$ of the atoms in the molecule by means of the equation 
TABLE III

Character Table a of $C_{s}$

\begin{tabular}{|c|c|c|c|}
\hline & $\begin{array}{l}\text { E } \\
\text { E }\end{array}$ & $\begin{array}{c}(23)^{*} \\
\sigma_{v}\end{array}$ & Operators $^{\mathrm{b}}$ \\
\hline$A^{\prime}$ & 1 & 1 & $J_{X}, J_{Y}, J_{Z} ; J_{c} ; \pi_{c} ; \mu_{a}, \mu_{b}$ \\
\hline$A^{\prime \prime}$ & 1 & -1 & $\mu_{X}, \mu_{Y}, \mu_{Z} ; J_{a}, J_{b} ; \pi_{a}, \pi_{b} ; \mu_{c}$ \\
\hline \multicolumn{4}{|c|}{$\begin{array}{l}{ }^{a} \text { The point-group operation } \sigma_{v} \text { corresponds to } \\
\text { the permutation-inversion operation }(23)^{*} \text { for } \\
\text { the acetaldehyde atom numbering in Fig. } 1 \text {. } \\
\text { bXYZ and abc are laboratory-fixed and molecule- } \\
\text { fixed axis systems. The molecular symmetry } \\
\text { plane is assumed to contain the a and b axes. }\end{array}$} \\
\hline
\end{tabular}

$$
\mathbf{R}_{i}=\mathbf{R}+S^{-1}(\chi \theta \phi)\left[\mathbf{a}_{i}+\mathbf{d}_{i}\right],
$$

where $\mathbf{R}$ represents the laboratory-fixed coordinates of the center of mass of the molecule, $\mathbf{a}_{i}$ represents the equilibrium position of atom $i$ in the molecule-fixed axis system, and $S(\chi \theta \phi)$ is the direction cosine matrix defined in Ref. (2). Table IV gives the transformation properties of the Eulerian angles, small-amplitude vibrational displacement vectors, and vibrational normal coordinates under the operations of $C_{s}$. These transformation properties lead to the symmetry species shown in Table III for molecule-fixed components of the total angular momentum $(\mathbf{J})$, vibrational angular momentum $(\pi)$, and electric dipole moment operator $(\mu)$.

We see from Table III that operators of the form $\pi_{a} J_{a}$ are now totally symmetric; i.e., they are totally symmetric with respect to the point group $C_{s}$. The complete vibration-rotation Hamiltonian $H_{\mathrm{vr}}(2)$, which contains these and other vibrationrotation operators, is also totally symmetric, so that the two symmetry species ( $A^{\prime}$ and $A^{\prime \prime}$ ) of $C_{s}$ give us a rigorous classification system for eigenfunctions of $H_{\mathrm{vr}}$.

Selection rules can again be found by considering Eq. (2). The species of components of the direction cosine matrix $\Phi$ and of molecule-fixed components of the dipole

TABLE IV

Transformation Properties ${ }^{\mathrm{a}}$ in $C_{s}$ of the Born-Oppenheimer Variables ${ }^{\mathrm{b}}$

\begin{tabular}{llllllll}
$\mathbf{R}$ & $x, \theta, \phi$ & $\mathbf{d}_{2}$ & $\mathbf{d}_{3}$ & $\mathbf{d}_{i}, \quad i \neq 2,3$ & $Q_{j=1-10}$ & $Q_{j=11-15}$ \\
\hline$\sigma_{v}=(23)^{*}$ & $-\mathbf{R}$ & $\pi-x, \pi-\theta, \pi+\phi$ & $\sigma_{v} \mathbf{d}_{3}$ & $\sigma_{v} \mathbf{d}_{2}$ & $\sigma_{v} \mathbf{d}_{i}, \quad i \neq 2,3$ & $+Q_{j=1-10}$ & $-Q_{j=11-15}$
\end{tabular}

The correspondence abc $\rightarrow$ zxy is assumed, so that $\sigma_{v}=\sigma_{v}(x z)$, and $\sigma_{v}\left[d_{x}, d_{y}, d_{z}\right]$ $=\left[d_{x},-d_{y}, d_{z}\right]$ for any vector $d$. The equivalent rotation $(\underline{10})$ for $\sigma_{v}$ is $c_{2}(y)$. The variables traditionally used in the molecular wave function, as found on the right of Eq. (3). For acetaldehyde, linear combinations of the $d_{i}$ (atom numbering as in $\mathrm{Fig} .1$ ) are used to form the normal coordinates $Q_{j}, j=1-10$ (species $A^{\prime}$ ) and $j=11-15$ (species $\left.A^{\prime \prime}\right)$. 
moment operator $\mu$ in $C_{s}$ are as given in Table III, so that the laboratory-fixed $Z$ component of the dipole moment operator $\mu_{Z}$ now belongs to the symmetry species $A^{\prime \prime}$. As is well known, we can use the species of the molecule-fixed components $\mu_{x}$, $\mu_{y}, \mu_{z}$ to determine vibrational selection rules, and the species of $\mu_{\%}$ to determine overall vibration-rotation selection rules.

For a molecule with no large-amplitude vibrations, symmetry elements of the point group can be placed in a one-to-one correspondence with symmetry elements of the Longuet-Higgins group (9) of feasible permutation-inversion operations. For the equilibrium geometry and atom numbering scheme for $\mathrm{CH}_{3} \mathrm{CHO}$ shown in Fig. 1, we find that $E \rightarrow E$ and $\sigma \rightarrow(23)^{*}$. Bunker (10) has introduced the concept of equivalent rotation to describe the effects of permutation-inversion operations on the Eulerian (rotational) angles. Using this terminology, we find that the equivalent rotation for $(23)^{*}$ is $C_{2}(c)$.

One might expect, because the character under $(23)^{*}$, and therefore under $C_{2}(c)$, is well defined, that the parity of $K_{c}$ would also retain a rigorous group-theoretical meaning. As is seen below, this is strictly true only if the vibrational symmetry of the vibration-rotation state is either purely ${ }^{\mathrm{v}} A^{\prime}$ or purely ${ }^{\mathrm{v}} A^{\prime \prime}$. Table $\mathrm{V}$ shows the relations between ${ }^{\mathrm{vr}} \Gamma$, ${ }^{\mathrm{v}} \Gamma$, ${ }^{\mathrm{r}} \Gamma$, and $K_{c}$ obtained for these two cases, where for clarity and emphasis, left superscripts are used to differentiate between vibrational $(v)$, rotational $(r)$, and vibration-rotation ( $v r)$ symmetry species $(\Gamma)$.

The parity of $K_{a}$ does not have an absolutely rigorous meaning, even for vibrationrotation states with purely " $A$ ' or $^{\mathrm{V}} A$ " vibrational symmetry, since $c$-type Coriolis interactions will mix states according to the selection rules ${ }^{\mathrm{v}} A^{\prime} \leftrightarrow{ }^{\mathrm{V}} A^{\prime}$ or ${ }^{\mathrm{v}} A^{\prime \prime} \leftrightarrow{ }^{\mathrm{v}} A^{\prime \prime}$, and $\Delta K_{a}= \pm 1, \Delta K_{c}=0$. Nevertheless, for a near-prolate rotor with a large $A$ rotational constant and only moderate Coriolis interactions, the absolute value of $K_{a}$ often remains an excellent approximate quantum number.

Consider now, with the help of Table VI, what is meant by an $a$-, $b$-, or $c$-type vibration-rotation transition. Table VI gives schematically eight "upper-state" vibra-

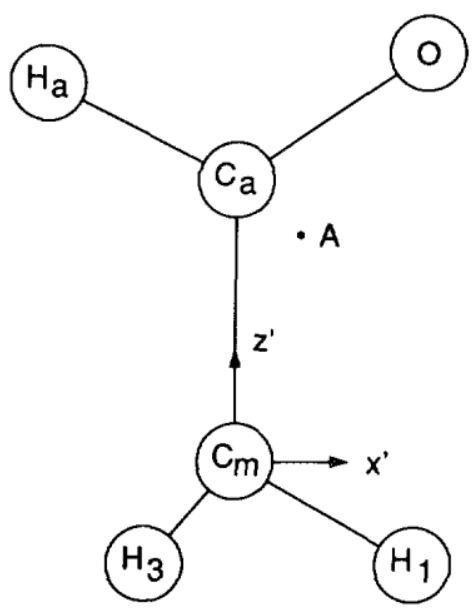

FIG. 1. Approximate structure and atom numbering scheme for the acetaldehyde molecule, $\mathrm{CH}_{3} \mathrm{CHO}$. In the $x^{\prime}, y^{\prime}, z^{\prime}$-axis system, which is used in Eq. (7), (i) the methyl carbon atom, $C_{m}$, lies at the origin, (ii) the $z^{\prime}$ axis lies along the $C_{3}$ axis of the methyl group, (iii) all atoms lie in the $x^{\prime} z^{\prime}$ plane, except for $\mathrm{H}_{3}$ and $\mathrm{H}_{2}$ (hidden by $\mathrm{H}_{3}$ in the figure), which lie symmetrically above and below the plane of the paper, respectively, and (iv) the center of mass of the molecule lies at the point $\mathbf{A}$ (near the aldehyde carbon $\mathrm{C}_{\mathbf{a}}$ ). 
TABLE V

Values of $K_{c}$ Corresponding to Values of $\left\langle(23)^{*}\right\rangle$ for Vibrational States of Pure $A^{\prime}$ or $A^{\prime \prime}$ Symmetry

\begin{tabular}{lcccc}
\hline $\operatorname{vr}_{\Gamma} a^{2}$ & $A^{\prime}$ & $A^{\prime}$ & $A^{\prime \prime}$ & $A^{\prime \prime}$ \\
$\langle(23) * b$ & +1 & +1 & -1 & -1 \\
$v_{\Gamma} \mathrm{b}$ & $A^{\prime}$ & $A^{\prime \prime}$ & $A^{\prime}$ & $A^{\prime \prime}$ \\
$r_{\Gamma} \mathrm{d}$ & $A^{\prime}$ & $A^{\prime \prime}$ & $A^{\prime \prime}$ & $A^{\prime}$ \\
$\mathrm{K}_{\mathrm{C}} \mathrm{e}$ & $\mathrm{e}$ & 0 & 0 & $\mathrm{e}$ \\
\hline
\end{tabular}

avibration-rotation symmetry of the wavefunction.

bxpectation value of $(23) *$ for this symmetry.

cSymetry species assumed for the vibrational

factor of the vibration-rotation wavefunction.

dSymmetry required for the rotational factor by

rows 1 and 3 .

eparity of $k_{c}$ required by row 4 (e=even, o=odd).

tion-rotation wavefunctions ( $\Psi_{\text {upper }}$ ), each of which consists of a main component $\left(\psi_{\text {unpert }}\right)$ plus components mixed in by $a$-type $\left(\psi_{\text {pert }}\left(\operatorname{Cor}_{a}\right)\right), b$-type $\left(\psi_{\text {pert }}\left(\operatorname{Cor}_{b}\right)\right)$, and $c$-type $\left(\psi_{\text {pert }}\left(\operatorname{Cor}_{c}\right)\right)$ Coriolis interactions. Each component is written as a simple product of a vibrational factor and a rotational factor. As mentioned earlier, the parity of $K_{c}$ remains a good quantum number only under Coriolis interactions which do not mix " $A$ ' and " $A$ " states. Immediately below the main component of each wavefunction is indicated the molecule-fixed dipole moment component $\left(\mu_{a}, \mu_{h}\right.$, or $\left.\mu_{c}\right)$ of the operator $\Phi_{\gamma_{i} \mu_{i}}$ in Eq. (2) which gives a nonvanishing transition moment from a lower state (chosen as an example) of the form $\psi_{\mathrm{v}} \psi_{\mathrm{r}}=\left|A^{\prime}\right\rangle\left|J_{\mathrm{ee}}\right\rangle$, where the subscripts e = even, o = odd on $J$ give the parity of $K_{a}, K_{c}$. The dipole moment component $\mu_{i}$ under each $\psi_{\text {unper }}$ gives rise to the usual terminology of $a$-type, $b$-type, $c$-type, or forbidden transition, and leads to the well-known result for a molecule like acetaldehyde with $z, x$, $y \rightarrow a, b, c$ that ${ }^{\mathrm{v}} A^{\prime} \leftrightarrow^{\mathrm{v}} A^{\prime}$ and ${ }^{\prime} A^{\prime \prime} \leftrightarrow^{\prime} A^{\prime \prime}$ transitions are induced by the $a$ and /or $b$ component of the dipole moment operator (an $a / b$ hybrid band (6)), while ${ }^{\mathrm{V}} A^{\prime \prime} \leftrightarrow$ ${ }^{\prime} A$ ' bands are induced by the $c$ component (a $c$-type band $(6)$ ).

Consider next the effects of Coriolis mixing on the transition moment for a nominal (main component) ${ }^{v} A^{\prime} \leftrightarrow^{v} A^{\prime} a / b$ hybrid band. We see from rows 2 and 4 of Table VI that $\mu_{h}$ and $\mu_{c}$ dipole matrix elements now contribute intensity to the nominal $a$ type lines, and that $\mu_{a}$ and $\mu_{c}$ matrix elements contribute intensity to the nominal $b$ type lines, but that no dipole moment components, not even $\mu_{c}$, contribute intensity to the nominal $c$-type transition $\left|A^{\prime}\right\rangle\left|J_{\mathrm{oe}}\right\rangle \leftrightarrow\left|A^{\prime}\right\rangle\left|J_{\mathrm{ee}}\right\rangle$ (see row 3). Also, no components give intensity to the forbidden transition $\left|A^{\prime}\right\rangle\left|J_{\mathrm{ec}}\right\rangle \leftrightarrow\left|A^{\prime}\right\rangle\left|J_{\mathrm{ee}}\right\rangle$ (see row 1 ).

Similar considerations for the effects of Coriolis interaction on a nominal " $A$ " $\leftrightarrow$ ' $A$ ' $c$-type band show that both $\mu_{a}$ and $\mu_{h}$ matrix elements contribute intensity to the nominal $c$-type lines, but neither contributes intensity to the nominal $a$-type or $b$ type transitions, i.e., to $\left|A^{\prime \prime}\right\rangle\left|J_{\mathrm{eo}}\right\rangle \leftrightarrow\left|A^{\prime}\right\rangle\left|J_{\mathrm{ec}}\right\rangle$ or $\left|A^{\prime \prime}\right\rangle\left|J_{\infty}\right\rangle \leftrightarrow\left|A^{\prime}\right\rangle\left|J_{\mathrm{ee}}\right\rangle$ transitions. On the other hand, all three transition moments contribute to the nominally forbidden $\left|A^{\prime \prime}\right\rangle\left|J_{\mathrm{ee}}\right\rangle \leftrightarrow\left|A^{\prime}\right\rangle\left|J_{\mathrm{ee}}\right\rangle$ transition 
Dipole Moment Components Giving Nonzero a Transition Moment Matrix Elements from $\Psi_{\text {lower }}=\left|A^{\prime}\right\rangle\left|J_{K_{a}=e, K_{\mathrm{r}}=e}\right\rangle$ to Coriolis-Perturbed ${ }^{\mathrm{b}} \Psi_{\text {upper }}$ Wavefunctions

$$
\begin{aligned}
& \Psi_{\text {upper }}=\psi_{\text {unpert }}+\psi_{\text {pert }}\left(\operatorname{Cor}_{a}\right)+\psi_{\text {pert }}\left(\operatorname{Cor}_{b}\right)+\psi_{\text {pert }}\left(\operatorname{Cor}_{c}\right) \\
& \left.\left.\left|v r_{A^{\prime}}\right\rangle=\left|v_{A^{\prime}}^{v}\right\rangle\left|J_{e e}\right\rangle+\left.\varepsilon_{a}\right|_{f} ^{v} A^{\prime \prime}\right\rangle\left|J_{e o^{\prime}}\right\rangle+\left.\varepsilon_{b}\right|_{A^{\prime \prime}}\right\rangle\left|J_{o o}\right\rangle+\left.\varepsilon_{c}\right|^{v} A^{\prime}>\left|J_{o e^{\prime}}\right\rangle
\end{aligned}
$$

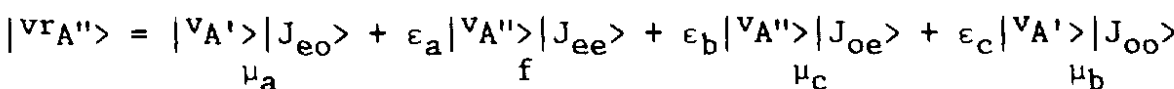

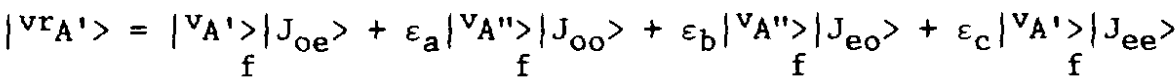

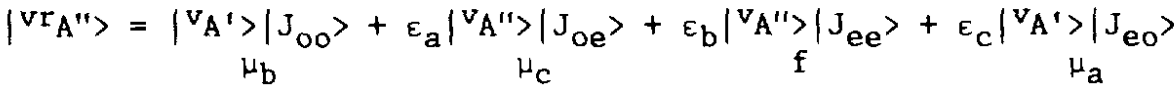

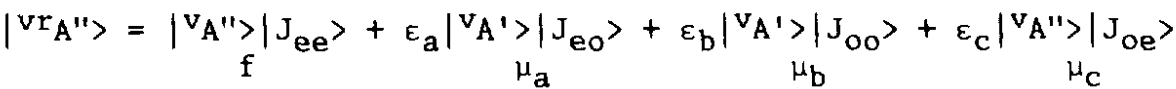

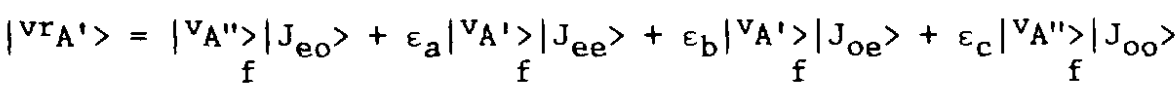

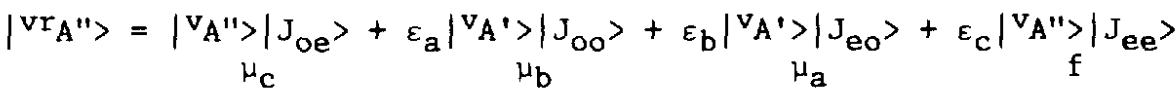

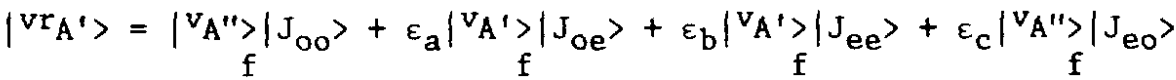

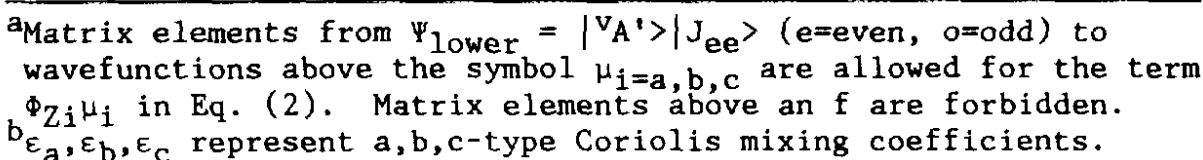

We have discussed the examples in Table VI in some detail to illustrate the ambiguities and confusion which arise when the nominal $a-, b$-, or $c$-type character of a transition does not coincide with the direction of the dipole moment component actually responsible for the major contribution to the transition moment matrix element. This discussion also serves as preparation for the similar but more complicated discussion of $a-, b$-, and $c$-type transitions when internal rotation splittings are present. In that case the torsion-rotation coupling $p_{\alpha} J_{z}$ plays a role analogous to the vibrationrotation Coriolis coupling here.

\section{Limiting Cases (vrt and $r$ ) with Permutation-Inversion Group $G_{6}$}

The first limiting case (vrt) is appropriate when small-amplitude vibrations ( $v$ ), overall rotation ( $r$ ), and internal rotation ( $t$ for torsion) are considered. The other case $(\mathrm{rt})$ is appropriate when only overall rotation and internal rotation are considered ( $\mathrm{rt}$ ). For both cases point groups can no longer be used (11), and one must change to a Longuet-Higgins permutation-inversion group $(9,10)$. For the problem at hand, this group contains six elements, which for acetaldehyde with the numbering scheme in Fig. 1 are $E,(123),(132),(12)^{*},(13)^{*}$, and (23)*. This permutation-inversion group, often called $G_{6}$, is isomorphic with the point group $C_{3 v}$, even though the atoms 
in acetaldehyde have an exceedingly low probability of passing through a $C_{3 v}$ configuration during their vibrational excursions. Because the symmetry species and character table notation of $C_{3 v}$ are so well known, however, it is customary for spectroscopists to simply adopt the notation for the isomorphic $C_{3 v}$ point group when dealing with the permutation-inversion group $G_{6}$. Table VII gives this character table and symmetry species.

Table VII indicates that wavefunctions of species $A_{1}$ transform into themselves, and wavefunctions of species $A_{2}$ transform into their negatives under the operation (23)*. This situation is similar to that described in the preceding section, with $A_{1}$ and $A_{2}$ here playing the role of $A^{\prime}$ and $A^{\prime \prime}$ there. The precise meaning of the $C_{3 v}$ symmetry operations is defined in Section 3; only a summary of the results obtained from those detailed considerations is presented now.

The variable transformations described in Section 3 can be applied to $J=K=0$ high-barrier torsional wavefunctions to show that they consist of an $A_{1}+E$ pair when $v_{t}=$ even, and an $A_{2}+E$ pair when $v_{t}=$ odd. (The $A_{1} / A_{2}$ alternation with $v_{l}$ is related to the fact that the small-amplitude torsional vibration $\nu_{15}$ in $\mathrm{CH}_{3} \mathrm{CHO}$ is antisymmetric with respect to the plane of symmetry and is thus of species $A^{\prime \prime}$ in the $C_{s}$ point group of the non-internally rotating molecule.) One can further show that rotational functions with $K_{c}=$ even and odd are of $A_{1}$ and $A_{2}$ symmetry species, respectively. Rotationtorsion levels of $A$ species ( $A_{1}$ or $A_{2}$ ) thus arise only from torsional levels of $A$ species, while rotation-torsion levels of $E$ species arise only from torsional levels of $E$ species.

It is tempting, for completeness, to include a discussion of the small-amplitude vibrations at this point, but such discussions can be quite complicated when a largeamplitude motion is present (12-14). This complication can be understood in the case of acetaldehyde by noting that the role of the "in-plane" methyl hydrogen is played by all three methyl hydrogens in turn during the internal rotation motion, making impossible any simple definition for a small-amplitude mode of the in-plane methyl hydrogen. Even though the vibration-rotation-torsion (vrt) case is not carefully

TABLE VII

Character Table ${ }^{\mathrm{a}}$ for $G_{6}=C_{3 v}$

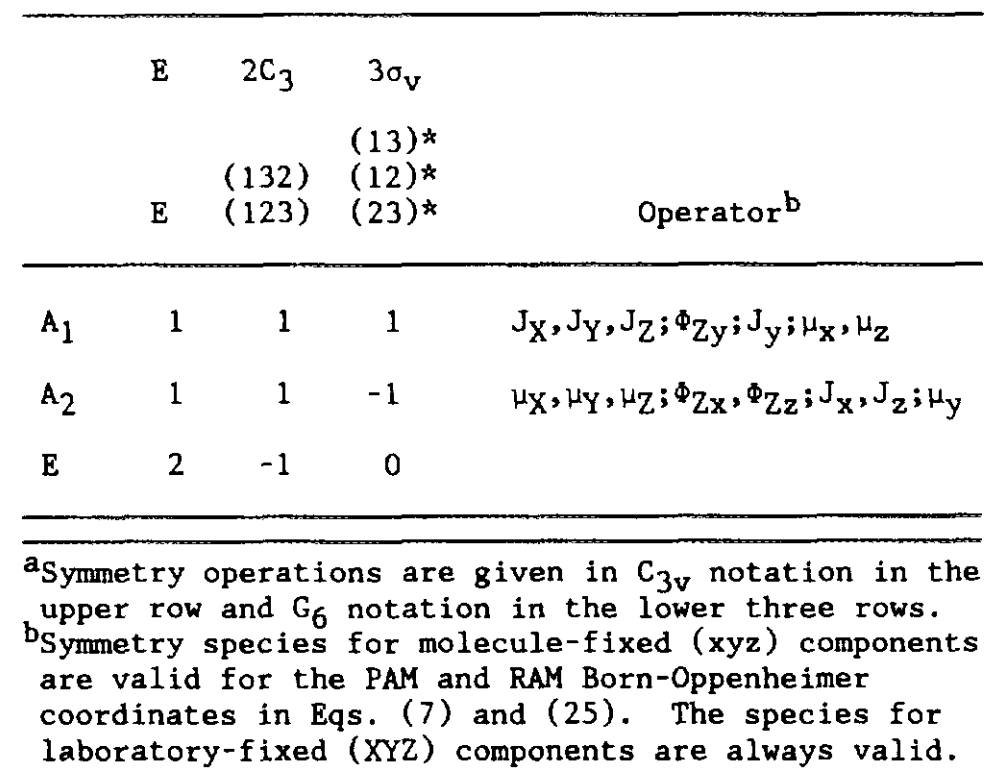


considered here, it seems likely that small-amplitude vibrational motions localized in the frame, which are of symmetry $A^{\prime}$ and $A^{\prime \prime}$ in $C_{s}$, can simply be considered as smallamplitude motions of $A_{1}$ and $A_{2}$ symmetry in $C_{3 v}$. We thus limit consideration in this paper to states with vibrational excitations localized in the frame (e.g., in the aldehyde group of acetaldehyde).

Consider now vibration-rotation-torsion states of symmetry species ${ }^{\mathrm{vn}} A_{1}$ and ${ }^{\mathrm{vr}} A_{2}$. We find from energy considerations that such levels are grouped into close-lying pairs of $K$-type doublets, one member of the pair having the symmetry species ${ }^{\mathrm{vrt}} A_{1}$, the other ${ }^{\mathrm{vn}} A_{2}$. Because the equivalent rotation for $(23)^{*}$ is $C_{2}(c)$ for the conformation shown in Fig. 1, the parity of $K_{c}$ can be defined by equating the \pm 1 group-theoretical character of $(23)^{*}$ to $(-1)^{K_{c}+v_{t}+\sum_{i} v_{i}\left(A^{\prime \prime}\right)}$, where $v_{l}$ is the torsional quantum number and $\sum_{i} v_{i}\left(A^{\prime \prime}\right)$ is a sum over all small-amplitude $A^{\prime \prime}$ vibrations. (As mentioned in the preceding paragraph, this expression is probably only valid when modes localized in the top are not excited.) Such a definition leads naturally to the traditional $J_{K_{a}, K_{c}}$ $=J_{K_{a}, J-K_{a}}$ and $J_{K_{a}, J-K_{a}+1}$ values, and ultimately to the result that no $c$-type transitions can occur (at least not until nuclear spin hyperfine interactions become important) involving ${ }^{\mathrm{vrt}} A_{1},{ }^{\mathrm{vrt}} A_{2}$ levels in a nominally $a, b$ hybrid band, and no $a$-type or $b$-type transitions can occur involving ${ }^{\mathrm{vrt}} A_{1},{ }^{\mathrm{vrt}} A_{2}$ levels in a nominally $c$-type band.

It is common in some parts of the literature to make use of principles similar to those in the paragraph above to introduce a + or - "parity" designation for rotationtorsion $A$ states. This label is unfortunately not a true parity, since the true parity describes transformation properties under the symmetry operation $E^{*}$, which does not happen to belong to the group $G_{6}$. Instead, these \pm labels are related in a slightly complicated way to the $A_{1}$ and $A_{2}$ species in $G_{6}$; namely, if the product ("parity") $\times(-1)^{J+v_{t}+\sum_{i} v_{i}\left(A^{\prime \prime}\right)}$ is positive or negative, the vibration-torsion-rotation symmetry species is $A_{1}$ or $A_{2}$, respectively. (The $J$ dependence of the phase factor arises from the behavior of the rotational wavefunction under $(23)^{*}$.) For vibrational transitions ${ }^{\mathrm{v}} A^{\prime} \leftrightarrow{ }^{\mathrm{v}} A^{\prime}$ and ${ }^{\mathrm{v}} A^{\prime \prime} \leftrightarrow{ }^{\mathrm{v}} A^{\prime \prime}$, the $G_{6}$ overall selection rules $A_{1} \leftrightarrow A_{2}$ then translates for this parity into $\pm \leftrightarrow \pm$ for $P$ and $R$ branches within a given $v_{t}$ level, or for $Q$ branches in a $\Delta v_{r}= \pm 1$ transition, or $\pm \leftrightarrow \mp$ for $Q$ branches within a given $v_{l}$ level, or $P$ and $R$ branches in a $\Delta v_{t}= \pm 1$ transition. These rules must be interchanged for ${ }^{\mathrm{v}} A^{\prime} \leftrightarrow^{\mathrm{v}} A^{\prime \prime}$ transitions. While $J$ is clearly a good quantum number for the isolated molecule, $v_{t}$ is a bad quantum number for energy levels lying near or above the top of the barrier. In such cases, the group-theoretical labels $A_{1}, A_{2}$ remain unambiguous; the \pm "parity" labels do not. (Again, the discussion above may not be valid when $\sum_{i} v_{i}\left(A^{\prime \prime}\right)$ includes vibrational excitations localized in the methyl top.)

Consider next wavefunctions of species ${ }^{\mathrm{vr}} E$. For such levels there is no energy grouping into nearly degenerate pairs. More importantly, however, the character for $(23)^{*}$ is zero, so that the expectation value for $(23)^{*}$, i.e., the diagonal elements of the $2 \times 2$ transformation matrix induced by $(23)^{*}$, must be equal and opposite for the two members of the degenerate $E$ state. (The magnitude of these diagonal elements can be adjusted to lie between zero and unity by suitable choice of linear combinations of the degenerate $E$ pair.) A value of zero for the character of $(23)^{*}$ implies that the significance of $K_{c}$ labels for ${ }^{\mathrm{vrt}} E$ functions (if such labels are used at all) must be based on something other than symmetry properties, and that $K_{\mathrm{c}}$ labels will not obey mathematically well-defined selection rules.

\section{$K_{c}$ Labels from Energy Ordering}

The most popular method for associating $K_{c}$ labels with rotational energy levels in internal rotor molecules is not based on considering symmetry properties of the levels 
as discussed above, but involves instead reasoning by analogy with the energy ordering of rotational levels in a rigid asymmetric top molecule. In this method, which we now examine in some detail, one simply requires that $K_{a}+1$ levels always lie above $K_{a}$ levels, and that $J_{K_{a}, J-K_{a}}$ levels always lie above $J_{K_{a}, J-K_{a}+1}$ levels.

In the moderate to high-barrier limit, such as that found for the $v_{t}=0$ and 1 levels in acetaldehyde, or the $v_{t}=0$ level of methanol, the torsional and symmetric top rotational contributions to the energy levels of a near-prolate top are approximately given by an equation of the form (15)

$$
F a_{1} \cos [(2 \pi / 3)(K \rho-\sigma)]+\bar{B} J(J+1)+(A-\bar{B}) K^{2},
$$

where approximate values of $F a_{1}=-1400 \mathrm{MHz}, \rho=\frac{1}{3}, \bar{B} \equiv(B+C) / 2=10000$ $\mathrm{MHz}$, and $A=56000 \mathrm{MHz}$ apply to the ground state of acetaldehyde (16), and a value of $F a_{1}=+35000 \mathrm{MHz}$ with $\rho, \bar{B}$, and $A$ unchanged can be obtained by refitting microwave and infrared data for the first torsional state given in Ref. (17). (Note that the sign convention for $\rho$ in Refs. (16,17) is opposite that in Ref. (15) and Eq. (4).) Rotational constants for both torsional states are such that asymmetric rotor $K$-type doubling increases approximately as $500 J(J+1) \mathrm{MHz}$ for $K=1$ levels.

Consider first rotation-torsion levels of ${ }^{\mathrm{rt}} A$ species. These have $\sigma=0$ in Eq. (4), and will occur as doubly degenerate ${ }^{\mathrm{r}} A_{1},{ }^{\mathrm{r}} A_{2}$ pairs for $K>0$, with wavefunctions of the approximate form

$$
\psi_{v^{\prime}, K_{a}, \sigma=0}(\alpha)\left[\left|J, K_{a}, M\right\rangle \pm\left|J,-K_{a}, M\right\rangle\right]
$$

where the torsional wavefunction $\psi(\alpha)$ depends on the $v_{l}, K_{a}$, and $\sigma$ quantum numbers. This pair of rotation-torsion wavefunctions will be split by the usual asymmetric rotor term $(1 / 2)(B-C)\left(J_{x}^{2}-J_{y}^{2}\right)$ and by the term $D_{a b}\left(J_{z} J_{x}+J_{x} J_{z}\right)$, and matrix elements of these operators in the basis functions of Eq. (5) will be modified by a torsional overlap integral $\left\langle\psi_{v_{i}^{\prime} K_{a, \sigma}^{\prime}=0} \mid \psi_{v_{i}, K_{a}, \sigma=0}\right\rangle$. In spite of these differences from the usual asymmetric rotor formalism, the rotational energy level pattern is not very different from that of an asymmetric rotor, and the labels $K_{a}, K_{c}$ can be attached to energyordered eigenfunctions in the usual way.

Consider next rotation-torsion levels of $E$ species, which have $\sigma= \pm 1$ in Eq. (4). There are actually two ${ }^{\pi} E$ levels for a given value of $K_{a}>0$, and each of these levels in turn is doubly degenerate. If only the energy contributions in Eq. (4) are considered, the four eigenfunctions for these two ${ }^{n} E$ levels correspond to the degenerate pair ( $\pm K$, $\sigma= \pm 1$ ) and ( $\mp K, \sigma= \pm 1$ ), respectively, where for convenience the first pair is often called the $+K$ level and the second pair the $-K$ level. The energy separation $\Delta E$ between the two $E$ states is calculated from Eq. (4) to be

$$
\begin{aligned}
\Delta E & =E(+K)-E(-K) \\
& =F a_{1}[\cos (2 \pi / 3)(K \rho-1)-\cos (2 \pi / 3)(K \rho+1)] \\
& =V\left(3 F a_{1} \sin (2 \pi \rho K / 3) .\right.
\end{aligned}
$$

Asymmetric rotor $K$-type doubling effects cannot split the degeneracy of these ${ }^{\pi} E$ states, but such effects can cause the two states to interact.

The energy ordering of $E$ levels in the first excited torsional state of $\mathrm{CH}_{3} \mathrm{CHO}$ is relatively simple to understand, because the large $v_{t}=1$ torsional splitting ( $\sqrt{ } 3 F a_{1}$ $=+60000 \mathrm{MHz}$ ) makes it possible to neglect $K$-type doubling contributions to the energy for $J$ values below 10. We then find from Eq. (6) that levels with $K_{c}=J$ $-K_{a}$ (the higher energy level of each $K$-type doublet) correspond to $( \pm K, \sigma= \pm 1)$ 
when $\sin (2 \pi \rho K / 3)$ is positive, but to $(\mp K, \sigma= \pm 1)$ when $\sin (2 \pi \rho K / 3)$ is negative. Figure 2, which displays $\sin (2 \pi \rho K / 3)$ for a value of $\rho=\frac{1}{3}$ as appropriate for $\mathrm{CH}_{3} \mathrm{CHO}$, shows that this sine function is positive for $K_{a}=1,2,3,4,10, \ldots$ and negative for $K_{a}=5,6,7,8,14, \ldots$

This same $K_{c}$ labeling scheme could also be applied to $E$ levels in the ground state. Here $F a_{1}$ is negative, so that levels with $K_{c}=J-K_{a}$ would correspond to $( \pm K$, $\sigma= \pm 1$ ) for just the opposite ranges of $K_{a}$.

For the ground state, however, the values of $\Delta E$ from Eq. (6) and the asymmetric rotor $K$-type doubling splittings are comparable in magnitude, and $K$-type doubling effects cannot be ignored. Instead, internal rotation splitting and $K$-type doubling compete to determine the mixing coefficients of basis set functions in the final eigenfunctions. This more complicated situation leads one to consider a method other than simple energy ordering for associating $K_{c}$ labels with rotational energy levels. This second method involves trying to define and determine how much even $K_{c}$ character and how much odd $K_{c}$ character is present in each wavefunction. The mathematical procedures needed to formalize such a procedure were beautifully set forth and applied by Alexander and Dagdigian (18) to the somewhat analogous problem of determining how much in-plane and out-of-plane $p$-orbital character is present in each $\Lambda$-doublet component of a rotating diatomic molecule in a ${ }^{2} \Pi$ state.

Since the procedures of Ref. ( 18 ) are based on symmetry operations, we must begin by defining the transformation properties of coordinates and wavefunctions under the operations of the $G_{6}$ molecular symmetry group. Before giving a table of transformation properties of the rotational, vibrational, and torsional (internal rotational) variables used in writing a wavefunction for acetaldehyde, it turns out to be necessary to clearly specify whether the internal rotation problem is being treated using a principal-axismethod (PAM) Hamiltonian (15), an internal-axis-method (IAM) Hamiltonian (15), or some intermediate method Hamiltonian $(19,20)$, since the coordinate systems used in these various Hamiltonians (often defined only implicitly following one or more contact transformations of the Hamiltonian operator) are quite different, giving rise to different boundary conditions in Schrödinger's differential equation (15), different molecular symmetry groups, and different transformation properties under

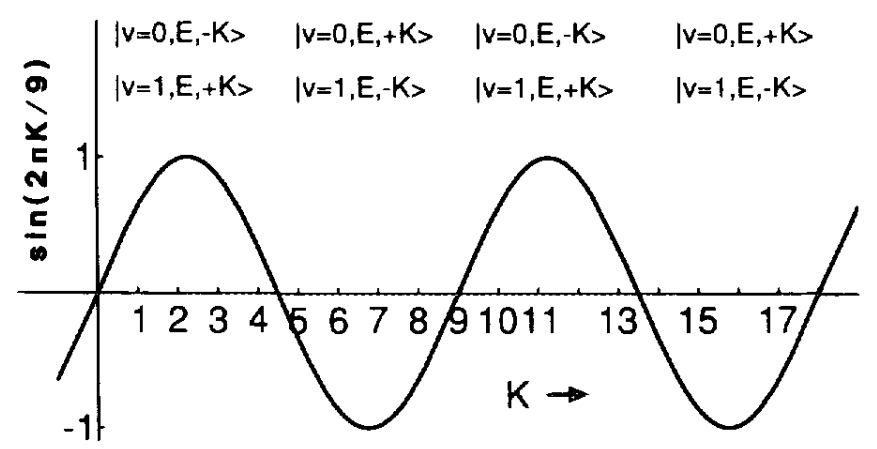

FIG. 2. Display of the sine function in Eq. (6), which governs the K-splitting of torsion-rotation levels of species $E$, drawn for a value of $\rho=\frac{1}{3}$, which is very close to the $\rho$ value for acetaldehyde. This sine function passes through zero at $K=0,4.5,9,13.5,18$, etc. The $|+K\rangle$ or $|-K\rangle$ wavefunction (for $\sigma=+1$ ) which would correspond to the $J_{K_{a}, J-K_{a}}$ function following traditional asymmetric rotor energy ordering considerations is shown for the $v=0$ and 1 torsional states in four regions of positive or negative sine function. 
the symmetry operations. (For example, in the next section we see that an $m$-fold extended group $G_{6}^{(m)}=C_{3 m, v}$ is required for the IAM treatment, whereas only the permutation-inversion group $G_{6}=C_{3 v}$ is required for the PAM treatment.)

\section{VARIOUS AXIS SYSTEMS FOR INTERNAL ROTATION PROBLEMS}

One of many ways of looking at the confusing variety of coordinate systems for treating internal rotation in molecules is as follows. Consider first a principal axis system which defines the axis labels $a, b, c$ in the molecule by the traditional spectroscopic moment-of-inertia ordering: $I_{a}<I_{b}<I_{c}$. We expect to find, in a molecular Hamiltonian written using an arbitrary $x, y, z$ molecule-fixed axis system, cross terms in $J_{x} J_{y}, J_{y} J_{z}$, and $J_{z} J_{x}$ arising from the failure to use a principal axis system and cross terms in $p_{\alpha} J_{x}, p_{\alpha} J_{y}$, and $p_{\alpha} J_{z}$ arising from Coriolis interactions between the internal rotation angular momentum $p_{\alpha}$ and the overall angular momentum. It turns out that by a suitable choice of contact transformation it is possible to eliminate some of these terms.

Let us now limit discussion to a molecule with a plane of symmetry containing the $a$ and $b$ inertial axes and the arbitrary $z$ and $x$ axes. Symmetry restrictions then eliminate the inertial cross terms $J_{x} J_{y}$ and $J_{y} J_{z}$, as well as the Coriolis cross term in $p_{\alpha} J_{y}$. If the inertial term $\left(I^{-1}\right)_{x z}\left(J_{x} J_{z}+J_{z} J_{x}\right)$ is eliminated by a contact transformation, the resulting Hamiltonian is referred to as a PAM Hamiltonian. If instead both the $p_{\alpha} J_{x}$ and the $p_{\alpha} J_{z}$ terms are eliminated, the resulting Hamiltonian is referred to as an IAM Hamiltonian. If only the $p_{\alpha} J_{x}$ term is eliminated, the resulting Hamiltonian has no name in the literature $(19,20)$; for reasons given below, we refer to it here as the rho-axismethod (RAM) Hamiltonian.

From the point of view of eliminating terms by suitable contact transformations, the existence of these various Hamiltonians is analogous to the existence of an $A$ reduction, an $S$-reduction, and intermediate reductions in Watson's treatment (3-5) of the ordinary asymmetric rotor centrifugal distortion Hamiltonian.

\section{PAM Coordinates and Hamiltonian}

It is convenient to summarize the principal axis method first because it is conceptually the simplest, both for deriving a Hamiltonian operator and for determining group-theoretical transformation properties of the coordinates. A PAM coordinate system for molecules with a methyl top rotor can be defined by the equation

$$
\mathbf{R}_{i}=\mathbf{R}+S^{-1}(\chi \theta \phi) \cdot T_{\mathrm{PAM}}^{-1}\left\{S_{i}^{-1}(\alpha 00) \cdot\left[\mathbf{a}_{i}+\mathbf{d}_{i}\right]-\mathbf{A}\right\},
$$

which can be understood as follows. The sum $\mathbf{a}_{i}+\mathbf{d}_{i}$ represents the equilibrium position and small-amplitude displacement vector for each atom, with vector components given in an $x^{\prime}, y^{\prime}, z^{\prime}$ axis system, where the $z^{\prime}$ axis is along the $C_{3}$ axis of the methyl top, and the center of mass of the molecule is at $\mathbf{A}$ (see Fig. 1). The rotation matrix $S_{i}^{-1}(\alpha 00)$, which does not occur in the analogous Eq. (3) for an ordinary molecule, is the unit matrix for atoms $i$ in the frame, but rotates the equilibrium positions and displacement vectors of atoms $i$ in the top through an angle $\alpha$ about the $z^{\prime}$ axis. The constant rotation matrix $T_{\mathrm{PAM}}^{-1}$, which also does not occur in Eq. (3), rotates the equilibrium positions (and displacements) of all atoms in the molecule, so that the final positions $T_{\mathrm{PAM}}^{-1}\left\{S_{i}^{-1}(\alpha 00) \cdot \mathbf{a}_{i}-\mathbf{A}\right\}$ are located in a principal inertial axis system $a, b, c$. (Note that $\mathbf{A}$ and $T_{\mathrm{PAM}}$ are constants because internal rotation of the symmetric top methyl group affects neither the center of mass nor the orientation 
of the principal axes of the molecule.) The rotation matrix $S^{-1}(\chi \theta \phi)$, containing the Eulerian angles, orients the whole molecule in the laboratory-fixed axis system, and the center-of-mass vector $\mathbf{R}$ translates the whole molecule to its final position in the laboratory.

If small-amplitude vibrations are neglected, as is commonly done, Eq. (7) can be used to derive the classical kinetic energy expression given in Eq. $(2-36)$ of (15). This in turn leads to the rotation-torsion Hamiltonian operator

$$
H_{\mathrm{PAM}}=F\left(p_{\alpha}-\boldsymbol{\rho} \cdot \mathbf{J}\right)^{2}+V(\alpha)+A J_{a}^{2}+B J_{b}^{2}+C J_{c}^{2}
$$

given in Eq. (2-38) of (15), which is valid for a molecule with a symmetric-top rotor, but with no other symmetry whatever. The rotational constants $A, B, C$, the internal rotation constant $F$, and the vector $\rho$ in this expression are related to the principal moments of inertia of the molecule $I_{a}, I_{b}, I_{c}$, and the moment of inertia of the methyl top $I_{\alpha}$, by the expressions

$$
\begin{array}{ll}
A=h^{2} / 2 I_{a} & \rho_{a}=\lambda_{a} I_{\alpha} / I_{a} \\
B=h^{2} / 2 I_{b} & \rho_{h}=\lambda_{b} I_{\alpha} / I_{b} \\
C=h^{2} / 2 I_{c} & \rho_{c}=\lambda_{c} I_{\alpha} / I_{c} \\
F=h^{2} / 2 r I_{\alpha} & r=1-I_{\alpha}\left(\lambda_{a}^{2} / I_{a}+\lambda_{h}^{2} / I_{b}+\lambda_{c}^{2} / I_{c}\right),
\end{array}
$$

where $\mathbf{J}$ is the total angular momentum, $p_{\alpha}$ is the angular momentum conjugate to the inertial rotation angle $\alpha, \lambda_{a}, \lambda_{b}, \lambda_{c}$ are direction cosines of the symmetry axis of the methyl group in the principal axis system of the molecule, and $V(\alpha)$ is the internal rotation potential energy function, which for a methyl rotor can always be expanded as a Fourier series in $\cos 3 n \alpha$.

We note, despite the use of principal axes, that if Eq. (8) is expanded and like powers of components of $\mathbf{J}$ are collected, the resulting expression (15) contains all three quadratic cross terms in $\left(J_{i} J_{j}+J_{j} J_{i}\right)$ and all three quadratic cross terms in $p_{\alpha} J_{i}$. Furthermore, the coefficient of $J_{i}^{2}$ is not simply $\hbar^{2} / 2 I_{i}$. Note also, that for the specific case of an acetaldehyde-like molecule, where the direction of the $C_{3}$ axis of the methyl rotor lies in the $a, b$ plane of symmetry of the molecule, that $\lambda_{c}=\rho_{c}=0$ in Eqs. (8) and (9), and $T_{\mathrm{PAM}}$ in Eq. (7) takes the simple form of a rotation about the $y$ axis; i.e., $T_{\mathrm{PAM}}=\mathrm{S}\left(0, \theta_{\mathrm{PAM}}, 0\right)$, for $a, b, c \rightarrow z, x, y$.

We now seek a set of transformations for the variables on the right side of Eq. (7) which will lead to the operations of the permutation-inversion group $G_{6}$ on the left side of Eq. (7). Since all elements of $G_{6}$ can be written as products of the form $\left[(23)^{*}\right]^{m}[(123)]^{n}$, where $0 \leqslant m \leqslant 1$ and $0 \leqslant n \leqslant 2$, it is sufficient to determine transformation properties for the variables under the two operations (123) and (23)*. It can be seen by direct substitution that the transformations given in Table VIII, when carried out on the right side of Eq. (7), lead to the desired permutation-inversion operation on the left.

\section{PAM Wavefunctions}

Matrix elements of the Hamiltonian in Eq. (8) are normally written in a basis set containing products of the type

$$
\left|e^{i(3 k+\sigma) k}\right\rangle|J, \pm K, M\rangle,
$$


TABLE VIII

Transformation Properties ${ }^{2}$ in $G_{6}$ of the PAM and RAM Born-Oppenheimer Variables ${ }^{\mathrm{b}}$

\begin{tabular}{|c|c|c|c|c|c|c|}
\hline & $\mathbf{R}$ & $x, \theta, \phi$ & $\alpha$ & $\mathbf{d}_{\mathrm{m}}$ & $\mathbf{d}_{2}$ & $\mathbf{d}_{1}, \quad i \neq c_{m}, 1,2,3$ \\
\hline (123) & $+R$ & $x, \theta, \phi$ & $\alpha+2 \pi / 3$ & $\mathrm{C}_{3} \mathrm{~d}_{\mathrm{C}} \mathrm{C}_{3} \mathrm{C}_{2}$ & $c_{3} d_{3} \quad c_{3} d_{1}$ & $+\mathbf{d}_{i}, \quad i \neq C_{m}, 1,2,3$ \\
\hline$(23) *$ & $-\mathbf{R}$ & $\pi-x, \pi-\theta, \pi+\phi$ & $-\alpha$ & $\sigma_{v} d_{C_{m}} \quad \sigma_{v} d_{1}$ & $\sigma_{v} d_{3} \quad \sigma_{v} d_{2}$ & $\sigma_{v} d_{1}, \quad i \neq c_{m}, 1,2,3$ \\
\hline
\end{tabular}

where the symmetric top wavefunctions $|J, \pm K, M\rangle\left(K=K_{a}\right.$ for acetaldehyde $)$ are assumed here to have the form and phases of normalized Wigner $D$ functions $\left[(2 J+1) / 8 \pi^{2}\right]^{1 / 2} D_{K M}^{(J)}(\chi \theta \phi)(2 I)$. The two factors of the basis function in Eq. (10) transform as follows under the two generating operations for $G_{6}$ given in Table VIII:

$$
\begin{aligned}
& (123)\left|e^{i(3 k+\sigma) \alpha}\right\rangle|J, \pm K, M\rangle=e^{+(2 \pi \sigma i / 3)}\left|e^{i(3 k+\sigma) \alpha}\right\rangle|J, \pm K, M\rangle \\
& (23)^{*}\left|e^{i(3 k+\sigma) \alpha}\right\rangle|J, \pm K, M\rangle=\left|e^{-i(3 k+\sigma) \alpha}\right\rangle(-1)^{J-K}|J, \mp K, M\rangle .
\end{aligned}
$$

Equations (11) indicate that the ${ }^{\mathrm{rt}} \Gamma=E$ basis set functions in Eq. (10) have been chosen such that $\left\langle(23)^{*}\right\rangle=0$. Because the Hamiltonian is totally symmetric under $G_{6}, E$-species eigenfunctions will also occur in pairs transforming according to Eqs. (11). In particular, $\left\langle(23)^{*}\right\rangle$ will be zero for all ${ }^{\pi} E$ eigenfunctions obtained in the present work.

We are now in a position to adapt Alexander and Dagdigian's procedure (18) to our problem. We consider an operator $C_{2}(c)$ which acts only on the Eulerian angles $\chi \theta \phi$ (and not on $\alpha$ ) so that

$$
C_{2}(c)\left[e^{i(3 k+\sigma) \alpha}\right]|J, \pm K, M\rangle=(-1)^{J-K}\left[e^{i(3 k+\sigma) \alpha}\right]|J, \mp K, M\rangle .
$$

Consider further the expectation values of $C_{2}(c)$ for a wavefunction which can be written as a product of a Wang rotational factor of the form

$$
\psi_{K \pm} \equiv 2^{-1 / 2}[|J, K, M\rangle \pm|J,-K, M\rangle]
$$

multiplied by some unspecified vibration-torsion factor $\psi_{\mathrm{v}}$. Since the expectation value for $C_{2}(c)$ gives by definition a value for $(-1)^{K c}$, we find

$$
\left\langle\psi_{\mathrm{v} 1} \psi_{K_{ \pm}}\left|C_{2}(c)\right| \psi_{\mathrm{v} t} \psi_{K_{ \pm}}\right\rangle= \pm(-1)^{J-K} \equiv(-1)^{K_{c}},
$$

which implies that $K_{c}=J-K$ for the sum and $K_{c}=J-K+1$ for the difference. Since, for the axis identification $a, b, c \rightarrow z, x, y$, for $K=K_{a}$, and for ordinary asymmetric rotor $K$-type doubling effects, the sum function in Eq. (13) always lies higher in energy than the difference, this definition for the parity of $K_{c}$ agrees with ordinary usage.

As demonstrated for the analogous problem in Ref. (18), this definition of the "parity of $K_{c}$ " can be extended to any linear combination of wavefunctions of the 
Expectation Values ${ }^{\mathrm{a}}$ of $(23)^{*}$ and $C_{2}(c)$. and Percentage of Even and Odd $K_{t}$ Character. ${ }^{b}$ for Various Torsion-Rotation Eigenfunctions of Acetaldehyde

\begin{tabular}{|c|c|c|c|c|c|c|c|c|c|c|c|c|c|c|c|c|c|}
\hline$v_{t}$ & $\mathrm{~J}$ & $\mathrm{~K}_{\mathrm{a}}$ & $\mathrm{K}_{\mathrm{c}}$ & $P^{c} \Gamma$ & $\left\langle(23)^{*}\right\rangle$ & $\left\langle\mathrm{C}_{2}(\mathrm{c})\right\rangle$ & $\%(\mathrm{e})$ & $\%(0)$ & $v_{t}$ & $\mathrm{~J} \mathrm{~K}$ & $\mathrm{~K}_{\mathrm{a}}{ }^{\mathrm{d}}$ & $\mathrm{K}_{\mathrm{c}}$ & $r$ & $\left\langle(23)^{*}\right\rangle$ & $\left\langle\mathrm{C}_{2}(\mathrm{c})\right\rangle$ & $\%(\mathrm{e})$ & $7(0)$ \\
\hline 0 & 0 & 0 & 0 & $+A_{1}$ & 1. & 1.000 & 100.00 & 0.00 & 0 & 0 & 0 & 0 & $\mathrm{E}$ & 0. & 1.000 & 100.00 & 0.00 \\
\hline 1 & 0 & 0 & 0 & $+A_{2}$ & -1 & 1.000 & 100.00 & 0.00 & 1 & 0 & 0 & 0 & $\mathrm{E}$ & 0. & 1.000 & 100.00 & 0.00 \\
\hline 0 & 1 & 0 & 1 & $+A_{2}$ & -1 & -1.000 & 0.00 & 100.00 & 0 & 1 & 0 & 1 & E & 0. & -1.000 & 0.00 & 100.00 \\
\hline 0 & 1 & 1 & 1 & $+\mathrm{A}$ & -1 & -0.989 & 0.55 & 99.45 & 0 & & -1 & 1 & $\mathrm{E}$ & 0. & -0.567 & 21.65 & 78.35 \\
\hline 0 & 1 & 1 & 0 & $-A_{1}$ & 1. & 0.989 & 99.45 & 0.55 & 0 & 1 & 1 & 0 & $\mathrm{~B}$ & 0. & 0.567 & 78.35 & 21.65 \\
\hline 1 & 1 & 0 & 1 & $+A_{1}$ & 1. & -1.000 & 0.00 & 100.00 & 1 & 1 & 0 & 1 & $\mathrm{E}$ & 0. & -0.998 & 0.10 & 99.90 \\
\hline 1 & 1 & 1 & 1 & $+A_{1}$ & 1. & -0.956 & 2.20 & 97.80 & 1 & $1-$ & -1 & 0 & $\mathrm{E}$ & 0. & 0.028 & 51.40 & 48.60 \\
\hline 1 & 1 & 1 & 0 & $-A_{2}$ & -1 & 0.956 & 97.80 & 2.20 & 1 & 1 & 1 & 1 & $\mathrm{E}$ & 0. & -0.030 & 48.50 & 51.50 \\
\hline 0 & 3 & 0 & 3 & $+A_{2}$ & -1 & -1.000 & 0.00 & 100.00 & 0 & 3 & 0 & 3 & $\mathrm{E}$ & 0. & -1.000 & 0.00 & 100.00 \\
\hline 0 & 3 & 1 & 3 & $+A_{2}$ & -1 & -0.989 & 0.55 & 99.45 & 0 & $3-$ & -1 & 3 & $\mathrm{E}$ & 0. & -0.962 & 1.90 & 98.10 \\
\hline 0 & 3 & 1 & 2 & $-A_{1}$ & 1. & 0.988 & 99.40 & 0.60 & 0 & 3 & 1 & 2 & E & 0. & 0.961 & 98.05 & 1.95 \\
\hline 0 & 3 & 2 & 2 & $-A_{1}$ & 1. & 0.956 & 97.80 & 2.20 & 0 & $3-2$ & -2 & 2 & $\mathrm{E}$ & 0. & 0.037 & 51.85 & 48.15 \\
\hline 0 & 3 & 2 & 1 & $+A_{2}$ & -1 & -0.956 & 2.20 & 97.80 & 0 & 3 & 2 & 1 & $\mathrm{E}$ & 0. & -0.037 & 48.15 & 51.85 \\
\hline 0 & 3 & 3 & 1 & $+A_{2}$ & -1 & -0.903 & 4.85 & 95.15 & 0 & $3-$ & -3 & 1 & $\mathrm{E}$ & 0. & -0.000 & 50.00 & 50.00 \\
\hline 0 & 3 & 3 & 0 & $-A_{1}$ & 1. & 0.903 & 95.15 & 4.85 & 0 & 3 & 3 & 0 & $\mathrm{E}$ & 0. & 0.000 & 50.00 & 50.00 \\
\hline 1 & 3 & 0 & 3 & $+A$ & 1. & -0.998 & 0.10 & 99.90 & 1 & 3 & 0 & 3 & $\mathrm{E}$ & 0. & -0.989 & 0.55 & 99.45 \\
\hline 1 & 3 & 1 & 3 & $+A_{1}$ & 1. & -0.956 & 2.20 & 97.80 & 1 & $3-1$ & -1 & 2 & $\mathrm{E}$ & 0. & 0.166 & 58.30 & 41.70 \\
\hline 1 & 3 & 1 & 2 & $-A_{2}$ & -1 & 0.953 & 97.65 & 2.35 & 1 & 3 & 1 & 3 & $\mathrm{E}$ & 0. & -0.177 & 41.15 & 58.85 \\
\hline 1 & 3 & 2 & 2 & $-A_{2}$ & -1 & 0.835 & 91.75 & 8.25 & 1 & $3-2$ & -2 & 1 & $\mathrm{E}$ & 0. & -0.001 & 49.95 & 50.05 \\
\hline 1 & 3 & 2 & 1 & $+A_{1}$ & 1. & -0.835 & 8.25 & 91.75 & 1 & 32 & 2 & 2 & $E$ & 0. & 0.000 & 50.00 & 50.00 \\
\hline 1 & 3 & 3 & 1 & $+A_{1}$ & 1. & -0.664 & 16. & 83 & 1 & $3-$ & -3 & 0 & $\mathrm{E}$ & 0. & -0.000 & 50.00 & 50.00 \\
\hline 1 & 3 & 3 & 0 & $-A_{2}$ & -1 & 0.664 & 83.20 & 16.80 & 1 & 3 & 3 & 1 & E & 0. & -0.000 & 50.00 & 50.00 \\
\hline
\end{tabular}

$a_{\left.<C_{2}(c)\right\rangle}$ is from an expression like Eq. (14) using numerical torsion-rotation eigenfunctions from the least squares fits of $v_{t}=0$ and 1 acetaldehyde data in Refs. $(16,17)$

The percent of even $(\%(e))$ and odd $(\%(o)) K_{c}$ character is determined from the expectation value of the operator $\mathrm{C}_{2}(\mathrm{c})$ such that: $\boldsymbol{Z}(\mathrm{e})+\%(\mathrm{o})=100$ and $\%(\mathrm{e})-\boldsymbol{Z}(0)=100\left\langle\mathrm{C}_{2}(\mathrm{c})\right\rangle$

The "parity" of the $A_{1}, A_{2}$ levels (see text).

The sign of $\mathrm{K}_{\mathrm{a}}$ in this column is taken from Refs. $(16,17)$, and is thus opposite to that described in the text associated with Eqs. (4)-(6) in this work. 
form given in Eq. (10). When the expectation value of $C_{2}(c)$ is +1 for a given linear combination, the parity of $K_{c}$ is even; when it is -1 , the parity of $K_{c}$ is odd. When the expectation value is exactly zero (as it is for either of the two functions in Eq. (10)), the wavefunction is a 50-50 mixture of even $K_{c}$ and odd $K_{c}$ functions. Table IX gives a number of expectation values for $C_{2}(c)$ and for $(23)^{*}$ obtained from actual eigenfunctions generated in our fits of $v_{t}=0$ and 1 microwave and infrared data for acetaldehyde $(16,17)$. (Even though these least-squares fit calculations were carried out in an RAM axis system (see below), expectation values for $(23)^{*}$ and $C_{2}(c)$ are the same as those for a PAM calculation, since the PAM and RAM systems for $\mathrm{CH}_{3} \mathrm{CHO}$ differ only by a rotation about the $c$ axis.)

\section{PAM Transition-Moment Matrix Elements}

Consider now the schematic matrix element of $\mu_{Z}$

$$
\begin{aligned}
{\left[c_{1}\left\langle{ }^{\mathrm{r}} A_{2}\left(K_{c}=0\right)^{\mathrm{t}} A_{1}\left(v_{t}=0\right)\right|+c_{2}\left\langle{ }^{\mathrm{r}} A_{1}\left(K_{c}=\mathrm{e}\right)^{\mathrm{t}} A_{2}\left(v_{t}=1\right)\right|\right] } \\
\left.\times\left.\left[\Phi_{Z_{a} \mu_{a}}+\Phi_{Z h \mu_{b}}+\Phi_{Z c} \mu_{c}\right]\right|^{\mathrm{r}} A_{1}\left(K_{c}=\mathrm{e}\right)^{\mathrm{t}} A_{1}\left(v_{t}=0\right)\right\rangle,
\end{aligned}
$$

representing an allowed ${ }^{\mathrm{r}} A_{2} \leftrightarrow{ }^{\mathrm{r}} A_{1}$ transition. The coefficients $c_{1}^{2}$ and $c_{2}^{2}$ correspond to the $\%(\mathrm{o})$ and $\%(\mathrm{e})$ entries in Table IX, respectively. Similar to the Coriolis mixing examples discussed in Table VI, nonzero matrix elements to the $c_{1}$ component can be induced by $\Phi_{Z a} \mu_{a}$ or $\Phi_{Z b} \mu_{b}$, while nonzero matrix elements to the $c_{2}$ component can be induced by $\Phi_{Z c} \mu_{c}$. If $c_{1}^{2} \gg c_{2}^{2}$, the transition would be classified as belonging to a $\Delta v_{1}=0 a, b$ hybrid band; if $c_{2}^{2} \gg c_{1}^{2}$, the transition would be classified as belonging to a $\Delta v_{t}=1 c$-type band.

Consider next the matrix element

$$
\begin{aligned}
{\left[c _ { 3 } \left\langle{ } ^ { \mathrm { r } } A _ { 1 } ( K _ { c } = \mathrm { e } ) ^ { \mathrm { t } } A _ { 1 } \left(v_{t}\right.\right.\right.} & \left.=0) \mid+c_{4}\left\langle{ }^{\mathrm{r}} A_{2}\left(K_{c}=\mathrm{o}\right)^{1} A_{2}\left(v_{t}=1\right)\right|\right] \\
& \left.\times\left.\left[\Phi_{Z a} \mu_{a}+\Phi_{Z b b} \mu_{b}+\Phi_{Z{ }^{\prime}} \mu_{c}\right]\right|^{\mathrm{r}} A_{1}\left(K_{c}=\mathrm{e}\right)^{\mathrm{t}} A_{1}\left(v_{t}=0\right)\right\rangle,
\end{aligned}
$$

representing a forbidden ${ }^{\pi} A_{1} \leftrightarrow{ }^{\pi} A_{1}$ transition. The coefficients $c_{3}^{2}$ and $c_{4}^{2}$ correspond to the $\%(\mathrm{e})$ and $\%(\mathrm{o})$ entries in Table IX. Again, similar to the examples in Table VI, matrix elements of $\Phi_{Z a} \mu_{a}, \Phi_{Z b} \mu_{b}$, and $\Phi_{Z_{i}} \mu_{c}$ to the $c_{3}$ and $c_{4}$ components are all zero, corresponding to the fact that no components of the dipole moment can make this grouptheoretically forbidden (in the absence of hyperfine mixings) transition allowed.

Consider finally the matrix element

$$
\begin{aligned}
& {\left[c_{5}\left\langle{ }^{\mathrm{r}} A_{2}\left(K_{c}=0\right)^{\mathrm{t}} E(\sigma=1)\right|+c_{6}\left\langle{ }^{\mathrm{r}} A_{1}\left(K_{c}=\mathrm{e}\right)^{\mathrm{t}} E(\sigma=1)\right|\right] } \\
&\left.\times\left.\left[\Phi_{Z a} \mu_{a}+\Phi_{Z b} \mu_{b}+\Phi_{Z c} \mu_{c}\right]\right|^{\mathrm{r}} A_{1}\left(K_{c}=\mathrm{e}\right)^{t} E(\sigma=1)\right\rangle,
\end{aligned}
$$

representing an allowed ${ }^{\mathrm{rt}} E \leftrightarrow{ }^{\mathrm{T}} E$ transition. The coefficients $c_{5}^{2}$ and $c_{6}^{2}$ correspond to the $\%(0)$ and $\%(\mathrm{e})$ entries in Table IX. Here we find that nonzero matrix elements to the $c_{5}$ component can be induced by $\Phi_{Z a} \mu_{a}$ or $\Phi_{Z b} \mu_{b}$, while nonzero matrix elements to the $c_{6}$ component can be induced by $\Phi_{Z c} \mu_{c}$. The transition would be classified as an $a, b$ hybrid or $c$-type line depending on the relative magnitudes of $c_{5}$ and $c_{6}$. Note that we have specified the $\sigma$ value, but not the $v_{t}$ value, of the torsional factors in the wavefunctions, because there is no group-theoretical distinction between torsional $E$ functions characterized by even and odd $v_{t}$ values. Further, it is not necessary to consider $\sigma=-1$ functions on the left of Eq. (17), because $\mu_{\%}$ obeys the strict selection rule $\Delta \sigma=0$ in the absence of hyperfine mixings. 
Table IX shows that ${ }^{~} \mathrm{E}$ states for acetaldehyde sometimes have $100 \%$ even or odd $K_{c}$ character, but at other times they are 50-50 mixtures of even and odd character. Qualitatively speaking, the parity of $K_{c}$ for ${ }^{n} E$ states is better defined when the effects of asymmetric rotor $K$-type doubling interactions dominate over the internal rotation splittings, e.g., in low- $K$, high- $J, v_{t}=0$ states. When internal rotation splittings dominate, as in high- $K$, low- $J, v_{l}=1$ states, the evenness or oddness of $K_{c}$ is essentially not defined at all.

As a final task in this section, we consider the laboratory-fixed $Z$ component of the dipole moment operator $\mu_{\chi}$ when the molecule-fixed components $\mu_{x}, \mu_{y}, \mu_{z}$ are expressed as a Fourier series in the internal rotation angle $\alpha$ and a Taylor series in the small-amplitude vibrational coordinates $Q_{i}$,

$$
\mu_{Z}=\Phi_{Z X X}(\chi \theta \phi) \mu_{x}\left(\alpha ; Q_{i}\right)+\Phi_{Z y}(\chi \theta \phi) \mu_{y}\left(\alpha ; Q_{i}\right)+\Phi_{Z z}(\chi \theta \phi) \mu_{z}\left(\alpha ; Q_{i}\right) .
$$

From the character table and transformation properties given in Tables VII and VIII, we see that $\mu_{Z}$ is of species $A_{2}$ in $G_{6}$, and the direction cosines $\Phi_{Z x}, \Phi_{Z y}, \Phi_{Z z}$ are of species $A_{2}, A_{1}, A_{2}$. This requires that the molecule-fixed dipole moment components $\mu_{x}, \mu_{y}, \mu_{z}$ be of species $A_{1}, A_{2}, A_{1}$, which is consistent with the fact that permanent moments (i.e., constants and therefore necessarily of species $A_{1}$ ) can exist along the $x$ and $z$ axes, but not along the $y$ axis.

We use below explicit symbols like $\mu_{x: i n}$ to indicate constant coefficients in the mixed Taylor ( subscript $\mathrm{i}$ ) and Fourier (subscript $n$ ) expansions, which are truncated after the linear terms in the Taylor series and after an arbitrary number of terms in the Fourier series. The transformation properties in Table VIII then require that the molecule-fixed dipole moment operators can be expressed as

$$
\begin{aligned}
\mu_{x}(\alpha, Q)=\mu_{x: 00}+ & \sum_{n} \mu_{x: 0 n}(1-\cos 3 n \alpha) \\
& +\sum_{i} Q_{i}\left[\mu_{x: i 0}+\sum_{n} \mu_{x: i n}(1-\cos 3 n \alpha)\right]+\sum_{j} Q_{j}\left[\sum_{n} \mu_{x: j n} \sin 3 n \alpha\right]
\end{aligned}
$$$$
\mu_{y}(\alpha, Q)=\sum_{n} \mu_{y ; 0 n} \sin 3 n \alpha+\sum_{i} Q_{i}\left[\sum_{n} \mu_{y ; i n} \sin 3 n \alpha\right]
$$$$
+\sum_{j} Q_{j}\left[\mu_{y ; j 0}+\sum_{n} \mu_{y: j n}(1-\cos 3 n \alpha)\right]
$$

$$
\begin{aligned}
\mu_{z}(\alpha, Q)= & \mu_{z ; 00}+\sum_{n} \mu_{z: 0 n}(1-\cos 3 n \alpha) \\
& +\sum_{i} Q_{i}\left[\mu_{z: i 0}+\sum_{n} \mu_{z ; i n}(1-\cos 3 n \alpha)\right]+\sum_{j} Q_{j}\left[\sum_{n} \mu_{z ; j n} \sin 3 n \alpha\right],
\end{aligned}
$$

where $\sum_{i}$ includes only the $A^{\prime}$ small-amplitude vibrations, and $\sum_{j}$ includes only the $A^{\prime \prime}$ small-amplitude vibrations. Again, a more careful treatment will probably be required when Eqs. (19) are used to derive results concerning small-amplitude vibrations localized in the top.

\section{Rho Axis Method}

The presence of the term $-2 F p_{a} \rho \cdot J$ in the PAM Hamiltonian of Eq. (8) makes it difficult to separate the torsional calculation from the rotational calculation. For this reason several investigators have preferred a slightly different coordinate axis system for numerical computational purposes, which is related to the principal $a, b, c$ axis 
system by a rotation chosen to eliminate the $-2 F p_{\alpha} \rho_{x} J_{x}$ and $-2 F p_{x} \rho_{y} J_{y}$ coupling terms; i.e., chosen so that the resultant torsional Hamiltonian operator has only $\Delta K$ $=0$ matrix elements. This rotation corresponds to making the new $z$ axis coincident with the $\rho$ vector, since then $\rho_{x}=\rho_{y}=0$ by definition. The formalism associated with this procedure has no name in the literature; for convenience we call it the rho axis method (RAM) here. In the case of an acetaldehyde-like molecule with an $a, b$ plane of symmetry and $a, b, c \rightarrow z, x, y$, the Coriolis cross term $p_{\alpha} J_{y}$ does not exist because of symmetry restrictions. A rotation about the $y$ axis, which we now examine in more detail, will eliminate the $p_{c} \rho_{x} J_{x}$ coupling term.

It can be shown by a somewhat tedious application of commutation relations that

$$
\begin{aligned}
e^{-i \theta J_{y} / \hbar}\left[J_{z} \pm i J_{x}\right] e^{+i \theta J_{y} / h} & =e^{ \pm i \theta}\left[J_{z} \pm i J_{x}\right] \\
e^{-i \theta J_{y} / h}\left[J_{y}\right] e^{+i \theta J_{y} / h} & =J_{y} \\
e^{-i \theta J_{y} / h}\left[\Phi_{Z z} \pm i \Phi_{Z x}\right] e^{+i \theta J_{y} / \hbar} & =e^{ \pm i \theta}\left[\Phi_{Z z} \pm i \Phi_{Z x}\right] \\
e^{-i \theta J_{y} / h}\left[\Phi_{Z y}\right] e^{+i \theta J_{y / h} / h} & =\Phi_{Z y y},
\end{aligned}
$$

with equations analogous to the last two for the $X$ and $Y$ components of the direction cosine matrix. Furthermore,

$$
e^{-i \theta J_{y} / \hbar}\left[f\left(\alpha, p_{\alpha}\right)\right] e^{+i \theta J_{y} / \hbar}=f\left(\alpha, p_{\alpha}\right) .
$$

While the simple rotation of Cartesian axes leading from the PAM to the RAM system is not normally discussed in this way, the first two of Eqs. (20) indicate that the desired rotation can be accomplished by the contact transformation

$$
H_{\mathrm{RAM}}=e^{-i \arctan \left(\rho_{x} / \rho_{z}\right) J_{y} / \hbar} H_{\mathrm{PAM}} e^{+i \arctan \left(\rho_{x} / \rho_{z}\right) J_{y} / \hbar},
$$

since

$$
e^{-\operatorname{iarctan}\left(\rho_{x} / \rho_{z}\right) J_{y} / \hbar}\left(p_{\alpha} \rho_{x} J_{x}+p_{\alpha} \rho_{z} J_{z}\right) e^{+\operatorname{iarctan}\left(\rho_{x} / \rho_{z}\right) J_{y} / \hbar}=\rho p_{\alpha} J_{z},
$$

where $\rho=\left(\rho_{x}^{2}+\rho_{z}^{2}\right)^{1 / 2}$. Note that the contact transformation in Eq. (22) does not involve the direction cosines $\lambda_{x}, \lambda_{z}$, which specify the orientation of the methyl top axis in the PAM axis system, but involves instead

$$
\theta_{\mathrm{RAM}} \equiv \arctan \left(\rho_{x} / \rho_{z}\right)=\arctan \left(I_{z} \lambda_{x} / I_{x} \lambda_{z}\right)
$$

(see Eqs. (9)). As a consequence, the RAM $z$ axis is parallel to the $C_{3}$ axis of the methyl top only when $I_{z}=I_{x}$.

This same conclusion can be obtained by using the last two of Eqs. (20) to transform the right-hand side of Eq. (7). The new relation between laboratory-fixed and moleculefixed coordinates becomes

$$
\begin{aligned}
\mathbf{R}_{i} & =\mathbf{R}+e^{-i \theta_{\mathrm{RAM}} J_{y} / h} S^{-1}(\chi \theta \phi) e^{+i \theta_{\mathrm{RAM}} J_{y} / h} \cdot T_{\mathrm{PAM}}^{-1} \cdot\left\{S_{i}^{-1}(\alpha 00) \cdot\left[\mathbf{a}_{i}+\mathbf{d}_{i}\right]-\mathbf{A}\right\} \\
& =\mathbf{R}+S^{-1}(\chi \theta \phi) \cdot S^{-1}\left(0,-\theta_{\mathrm{RAM}}, 0\right) \cdot T_{\mathrm{PAM}}^{-1} \cdot\left\{\mathbf{S}_{i}^{-1}(\alpha 00) \cdot\left[\mathbf{a}_{i}+\mathbf{d}_{i}\right]-\mathbf{A}\right\} \\
& =\mathbf{R}+S^{-1}(\chi \theta \phi) \cdot T_{\mathrm{RAM}}^{-1} \cdot\left\{S_{i}^{-1}(\alpha 00) \cdot\left[\mathbf{a}_{i}+\mathbf{d}_{i}\right]-\mathbf{A}\right\},
\end{aligned}
$$

which leads to an equation formally identical to Eq. (7) except that the rotation matrix $T_{\mathrm{PAM}}$ has been replaced by $T_{\mathrm{RAM}}$. The presence of $T_{\mathrm{RAM}}^{-1}$ indicates that the $z$ axis of the RAM system is nearly parallel to the $C_{3}$ axis of the methyl top only when $T_{\text {RAM }}$ represents a small rotation. The last of Eqs. (25) also shows that the meaning of the 
Eulerian angles $\chi \theta \phi$ has changed as expected; they now describe the orientation in space of the RAM axis system. (Note that by definition (2) the laboratory-fixed directions $X, Y, Z$ label the rows, and the molecule-fixed directions $x, y, z$ label the columns, of the direction cosine matrix $S^{-1}(\chi \theta \phi)$, so that atom positions in the $x, y$, $z$ system (ignoring the $\mathbf{d}_{i}$ ) are given by $T_{\mathrm{RAM}}^{-1} \cdot\left[S_{i}^{-1}(\alpha 00) \cdot \mathbf{a}_{i}-\mathbf{A}\right]$ in Eq. (25) and by $T_{\mathrm{PAM}}^{-1} \cdot\left[S_{i}^{-1}(\alpha 00) \cdot \mathbf{a}_{i}-\mathbf{A}\right]$ in Eq. (7).)

The full RAM torsion-rotation Hamiltonian is given by

$$
\begin{aligned}
H_{\mathrm{RAM}}=F\left(p_{\alpha}-\rho J_{z}\right)^{2}+V(\alpha)+A & \left(\cos \theta_{\mathrm{RAM}} J_{z}-\sin \theta_{\mathrm{RAM}} J_{x}\right)^{2} \\
& +B\left(\sin \theta_{\mathrm{RAM}} J_{z}+\cos \theta_{\mathrm{RAM}} J_{x}\right)^{2}+C J_{y}^{2} .
\end{aligned}
$$

The advantage of this Hamiltonian for computations arises from the fact that all operators containing the torsional angle $\alpha$ or its conjugate momentum are diagonal in the rotational quantum number $K$; all operators off-diagonal in $K$ arise from the purely rotational part of the problem. Numerical computations can then be divided into a torsional part and a rotational part. First, a set of torsional calculations, one for each $K$ value, is carried out using a relatively large torsional basis set. These calculations are presumed to involve interaction energies much larger than rotational energies ( either because the moment of inertia of the methyl top is much smaller than the moments of inertia of the whole molecule or because the torsional barrier is much higher in energy than typical rotational spacings). This basis set is then significantly reduced in size by discarding all but the lowest several torsional eigenfunctions for given $K$. Finally, the torsional eigenfunctions retained are prepared for the rotational calculation, involving all $2 J+1$ values of $K$ for given $J$, by multiplying them by the appropriate symmetric top rotational function $|J, K, M\rangle$.

The coordinate relations in Eqs. (25) indicate that the group-theoretical transformations given in Table VIII for $\chi \theta \phi$ and $\alpha$ remain formally unchanged in the RAM basis set. The dipole moment operator is thus again given by expressions having the form of Eqs. (18) and (19).

Note that because the $x$ and $z$ axes do not have the same meaning in the PAM and RAM systems, the coefficients $\mu_{x ; i n}$ and $\mu_{z ; i n}$ must be subjected to a rotation about the $y$ axis through the angle $\arctan \left(\rho_{x} / \rho_{z}\right)$ when comparing relative intensity contributions along different axes calculated in the two systems. Figure 3 shows the approximate orientations of the PAM $z$ axis and the RAM $z$ axis in the symmetry plane of acetaldehyde. Note that for this molecule, because of the great difference in magnitude of the $A$ and $B$ rotational constants, the RAM $z$ axis is more nearly parallel to the principal $a$ axis than to the $C_{3}$ symmetry axis of the methyl group.

\section{Internal Axis Method}

The internal axis method for treating the problem of overall rotation and internal rotation, which we again discuss from the point of view of contact transformations, is more difficult to understand in depth than the principal axis method. As described by Lin and Swalen in their classic review article (15) the historical development of the subject by Dennison, Hecht, and co-workers (22-25) led spectroscopists to perform three contact transformations in succession in order to pass from the PAM to the IAM Hamiltonian. We write these here as only two contact transformations, indicated symbolically by

$$
\begin{aligned}
H_{\text {IAM }} & =e^{-i S_{2}} e^{-i S_{1}} H_{\mathrm{PAM}} e^{+i S_{1}} e^{+i S_{2}} \\
& =e^{-i S_{2}} H_{\mathrm{RAM}} e^{+i S_{2}},
\end{aligned}
$$




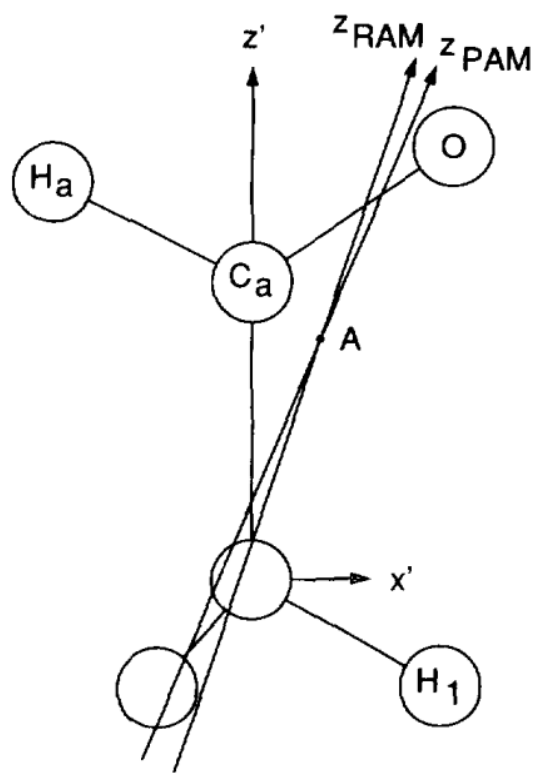

FIG. 3. Orientation in acetaldehyde of the methyl top axis $z^{\prime}$, the principal axis $z_{\mathrm{PAM}}$, and the $z_{\mathrm{RAM}}$ axis of $H_{\mathrm{RAM}}$ in Eq. (26). As can be seen from Eqs. (22) and (24), $z_{\mathrm{RAM}} \rightarrow z_{\mathrm{PAM}}$ as $\left(I_{z} / I_{x}\right) \rightarrow 0$, whereas $z_{\mathrm{RAM}}$ becomes parallel to $z^{\prime}$ as $\left(I_{z} / I_{x}\right) \rightarrow 1$, where $I_{z}$ and $I_{x}$ are the two principal moments of inertia in the symmetry plane of acetaldehyde in its equilibrium configuration.

where the first contact transformation is just that given in Eq. (22).

The purpose of the second contact transformation is to remove the term $-2 F \rho p_{\mathrm{a}} J_{z}$ remaining in the RAM Hamiltonian given in Eq. (26). Physically speaking, this is accomplished by rotating the whole molecule backward about the $\rho$ direction (the $z$ direction after the first contact transformation in Eq. (27)), which generates an angular momentum along the methyl top symmetry axis direction ( $\lambda$ in Eqs. (9)). The amount of rotation is chosen to just cancel the angular momentum generated by the methyl top internal rotation, thus removing in some sense the physical cause of the Coriolislike $-2 F \rho p_{\alpha} J_{z}$ interaction term. Mathematically, it can be seen relatively easily that

$$
\begin{aligned}
e^{-i \alpha \rho J_{z} / h}\left[p_{\alpha}-\rho J_{z}\right] e^{+i \alpha \rho J_{z} / h} & =p_{\alpha} \\
e^{-i \alpha \rho J_{z} / h}[f(\alpha)] e^{+i \alpha \rho J_{z} / h} & =f(\alpha) \\
e^{-i \alpha \rho J_{z} / h}\left[J_{x} \pm i J_{y}\right] e^{+i \alpha \rho J_{z} / \hbar} & =e^{ \pm i \alpha \rho}\left[J_{x} \pm i J_{y}\right] \\
e^{-i \alpha \rho J_{z} / h}\left[J_{z}\right] e^{+i \alpha \rho J_{z} / h} & =J_{z},
\end{aligned}
$$

leading us to write

$$
\begin{aligned}
H_{\mathrm{IAM}}= & e^{-i \alpha \rho J_{z} / \hbar} H_{\mathrm{RAM}} e^{+i \alpha \rho J_{z} / h}=F p_{\alpha}^{2}+V(\alpha) \\
& +A\left[+\cos \theta_{\mathrm{RAM}} J_{z}-\sin \theta_{\mathrm{RAM}}\left(\cos \rho \alpha J_{x}-\sin \rho \alpha J_{y}\right)\right]^{2}+B\left[+\sin \theta_{\mathrm{RAM}} J_{z}\right. \\
& \left.+\cos \theta_{\mathrm{RAM}}\left(\cos \rho \alpha J_{x}-\sin \rho \alpha J_{y}\right)\right]^{2}+C\left(\sin \rho \alpha J_{x}+\cos \rho \alpha J_{y}\right)^{2}, \quad \text { (29) }
\end{aligned}
$$

which can be shown with some effort to be equivalent to Eq. (2-31) of Ref. (15) with $a, b, c$ and $x, y, z$ (there) $\rightarrow-y, x, z$ and $-c, b, a$ (here), respectively. The transformed coordinate equation analogous to Eq. (25) becomes 


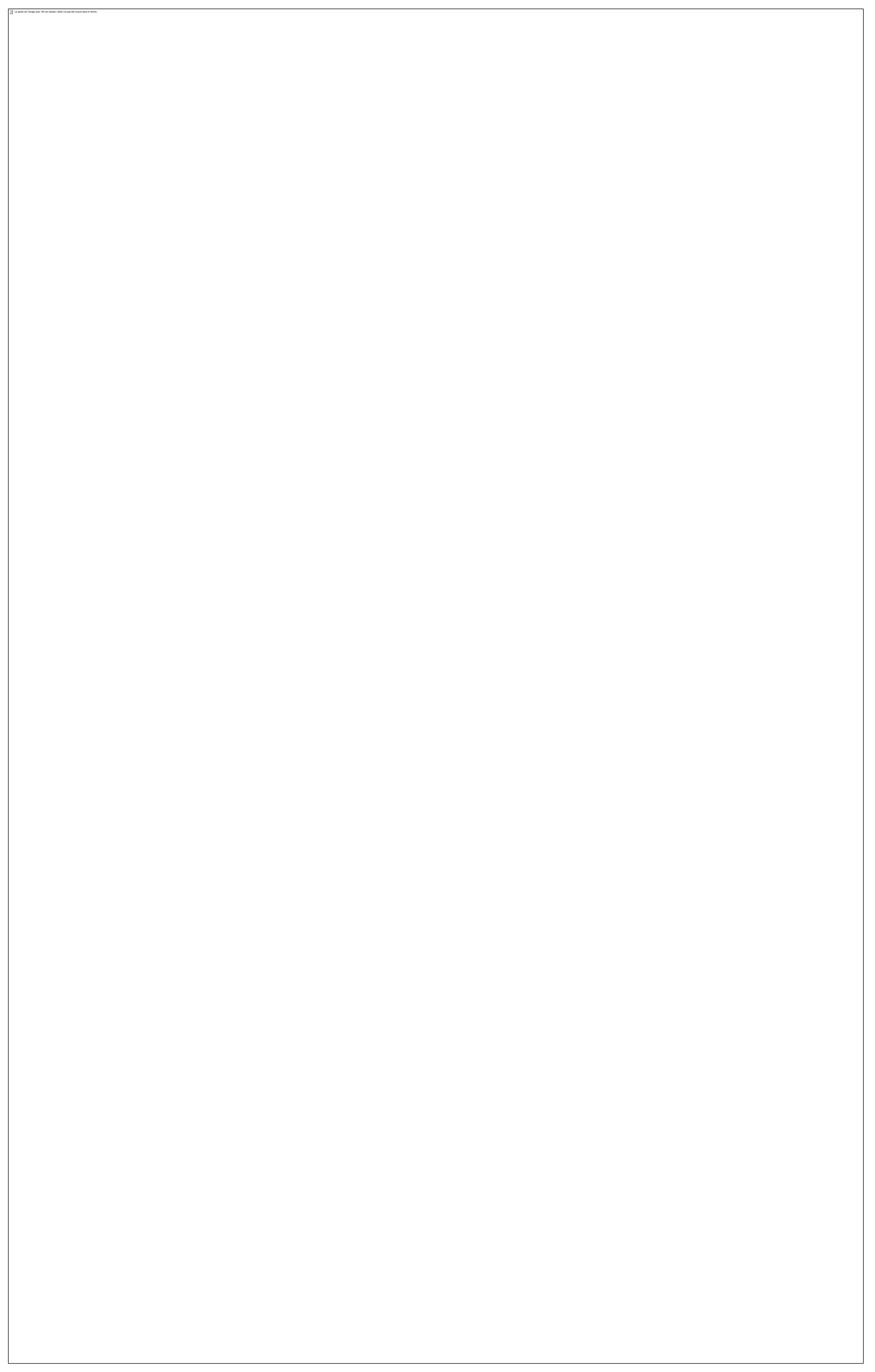




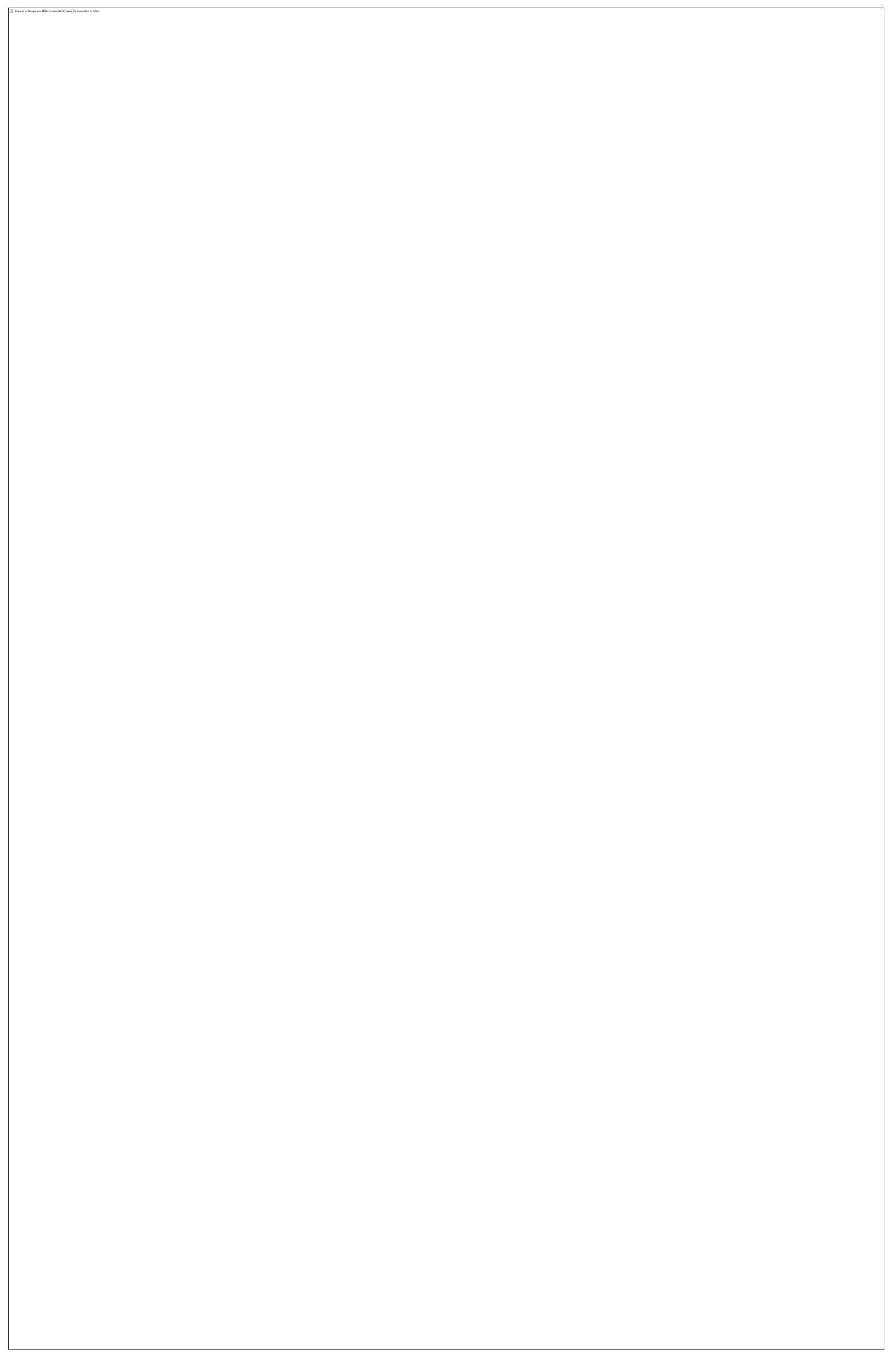




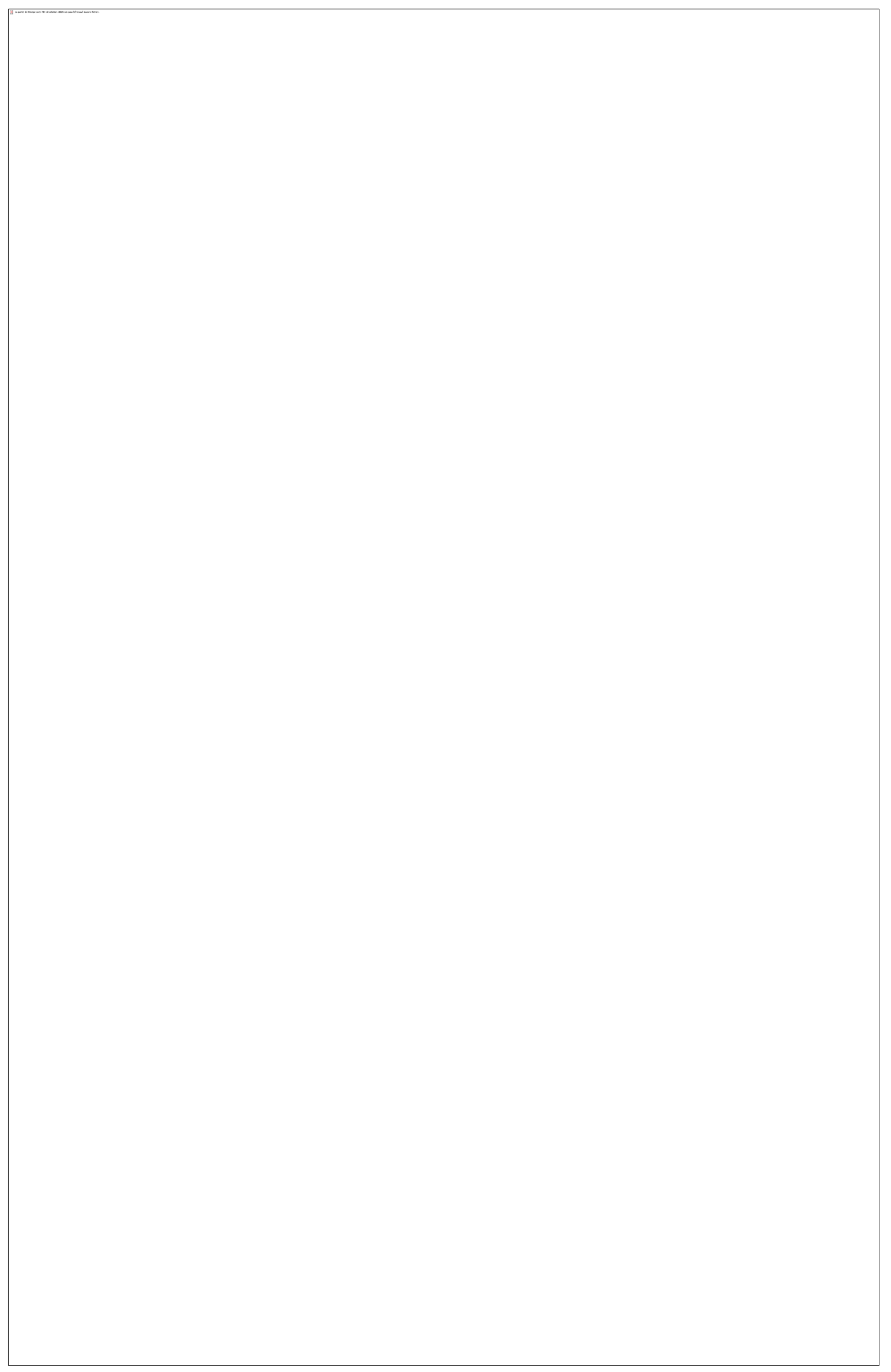




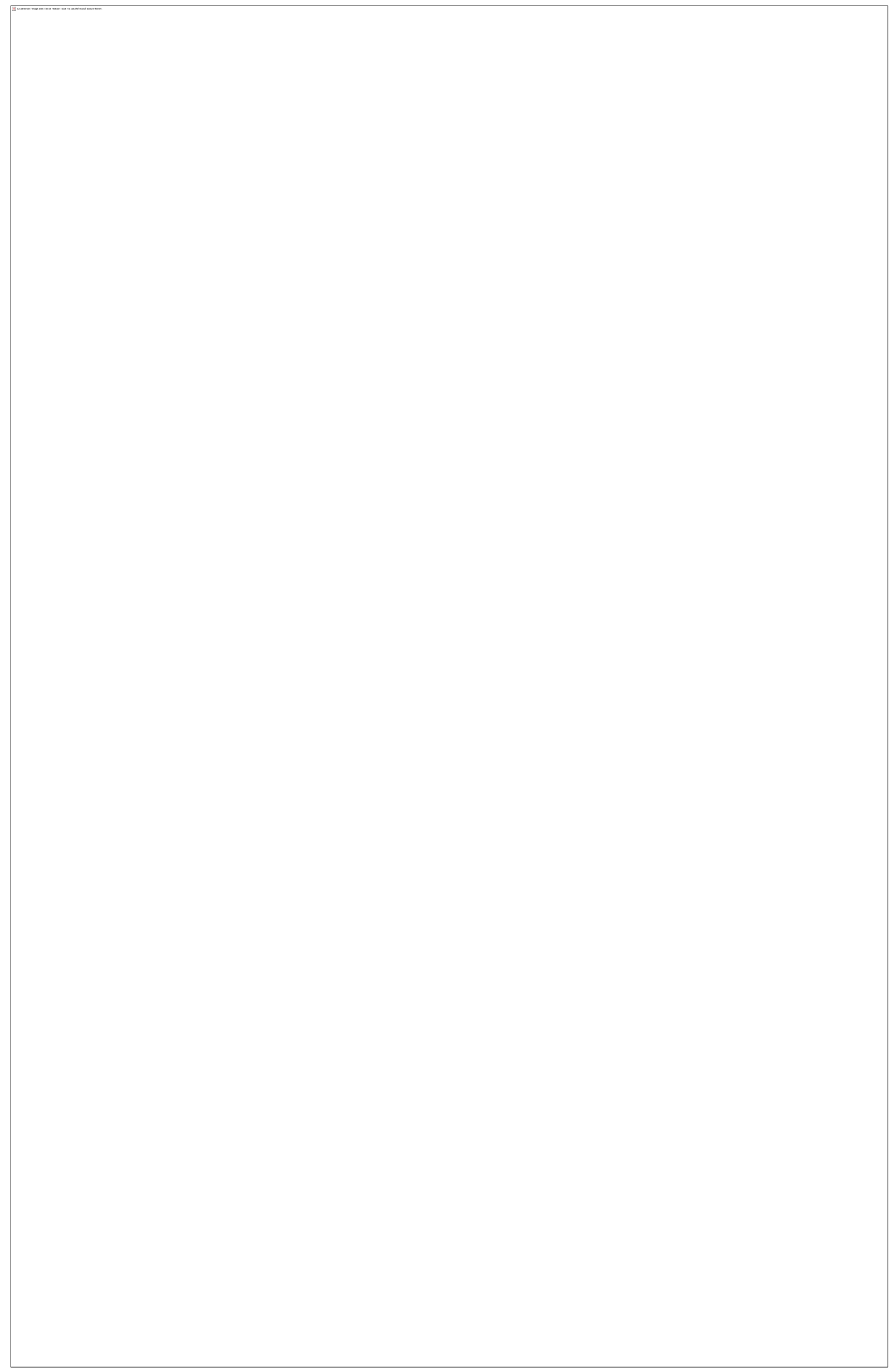




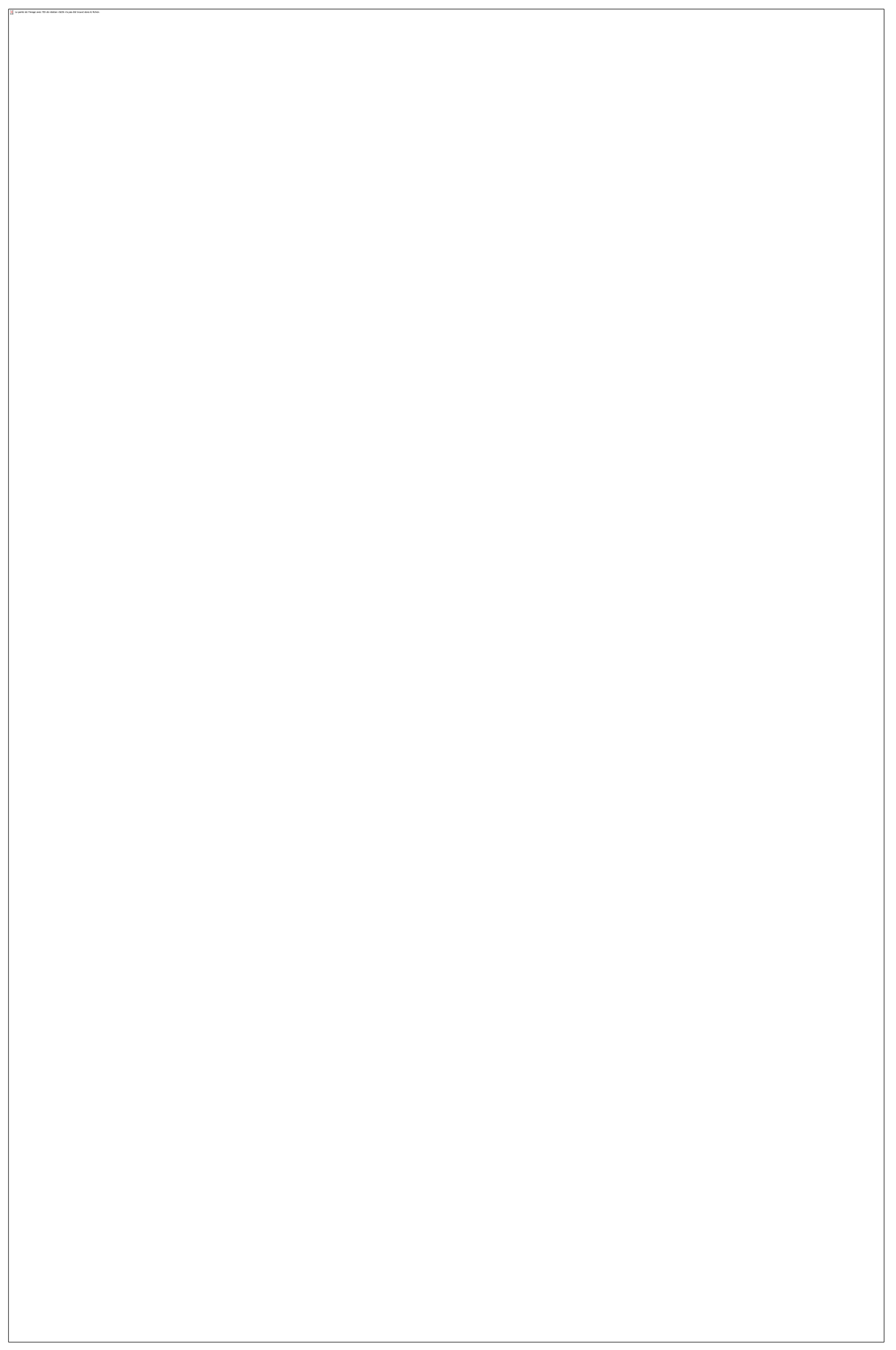




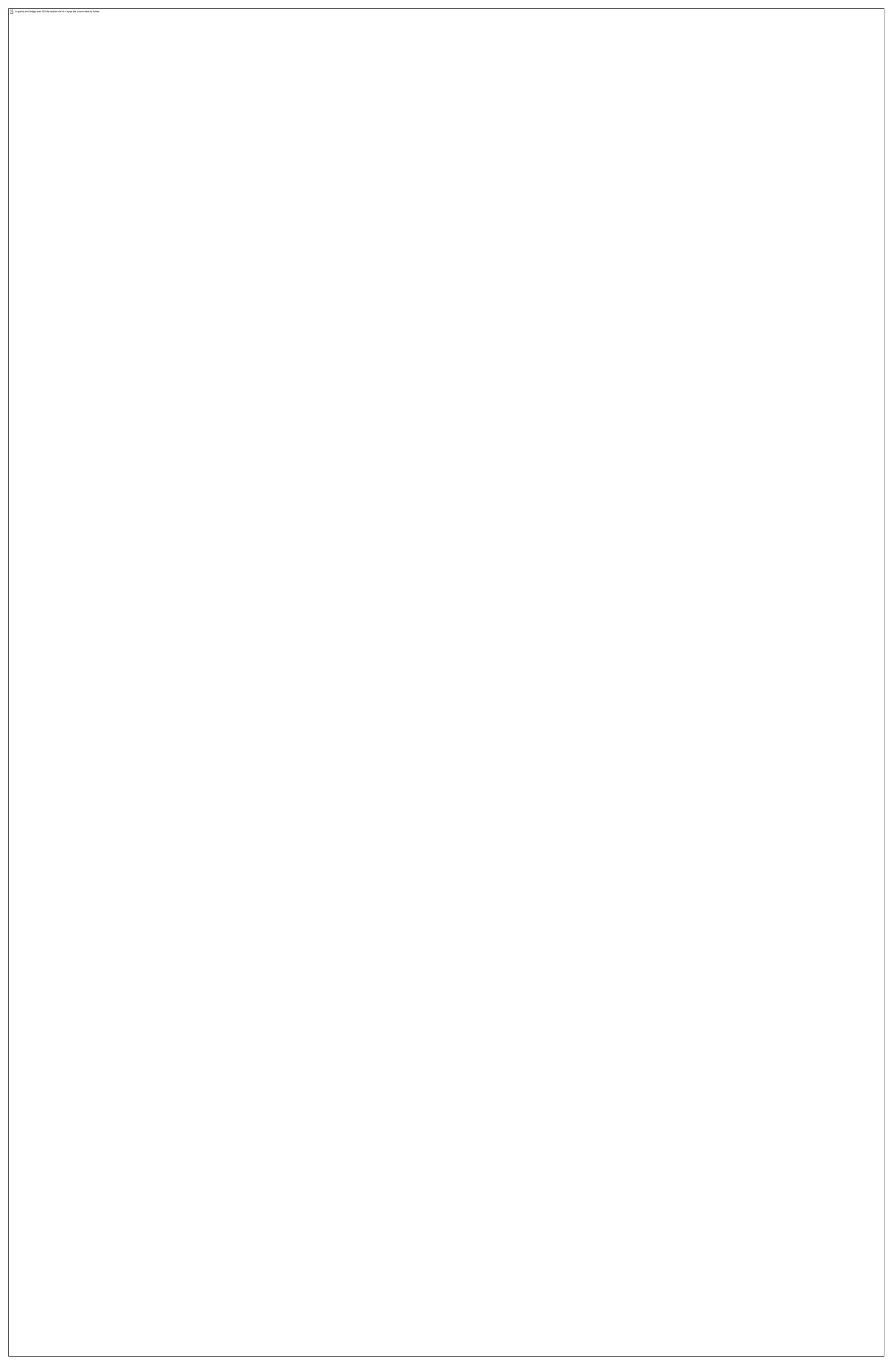




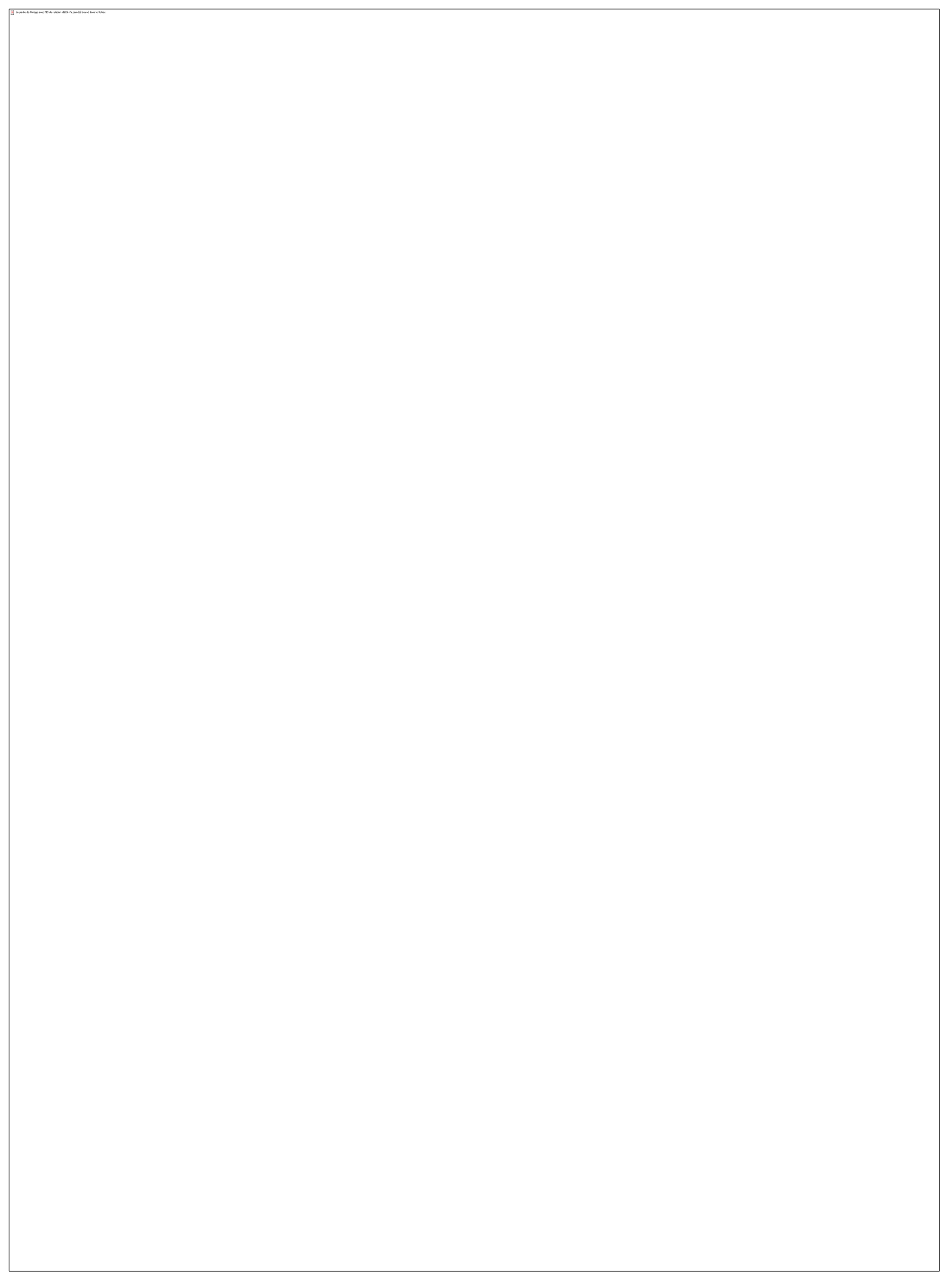

